\title{
Aerodynamics of inclined cylindrical bodies free-flying in a hypersonic flowfield
}

\author{
Patrick M. Seltner ${ }^{1}\left[\right.$ ] Sebastian Willems ${ }^{1} \cdot$ Ali Gülhan ${ }^{1} \cdot$ Eric C. Stern ${ }^{2} \cdot$ Joseph M. Brock $^{3} \cdot$ Michael J. Aftosmis $^{4}$
}

Received: 30 March 2021 / Revised: 13 July 2021 / Accepted: 15 July 2021

(c) The Author(s) 2021

\begin{abstract}
The influence of the flight attitude on aerodynamic coefficients and static stability of cylindrical bodies in hypersonic flows is of interest in understanding the re/entry of space debris, meteoroid fragments, launch-vehicle stages and other rotating objects. Experiments were therefore carried out in the hypersonic wind tunnel H2K at the German Aerospace Center (DLR) in Cologne. A free-flight technique was employed in $\mathrm{H} 2 \mathrm{~K}$, which enables a continuous rotation of the cylinder without any sting interferences in a broad angular range from $0^{\circ}$ to $90^{\circ}$. A high-speed stereo-tracking technique measured the model motion during free-flight and high-speed schlieren provided documentation of the flow topology. Aerodynamic coefficients were determined in careful post-processing, based on the measured 6-degrees-of-freedom (6DoF) motion data. Numerical simulations by NASA's flow solvers Cart3D and US3D were performed for comparison purposes. As a result, the experimental and numerical data show a good agreement. The inclination of the cylinder strongly effects both the flowfield and aerodynamic loads. Experiments and simulations with concave cylinders showed marked difference in aerodynamic behavior due to the presence of a shock-shock interaction (SSI) near the middle of the model.
\end{abstract}

\section{Graphic abstract}

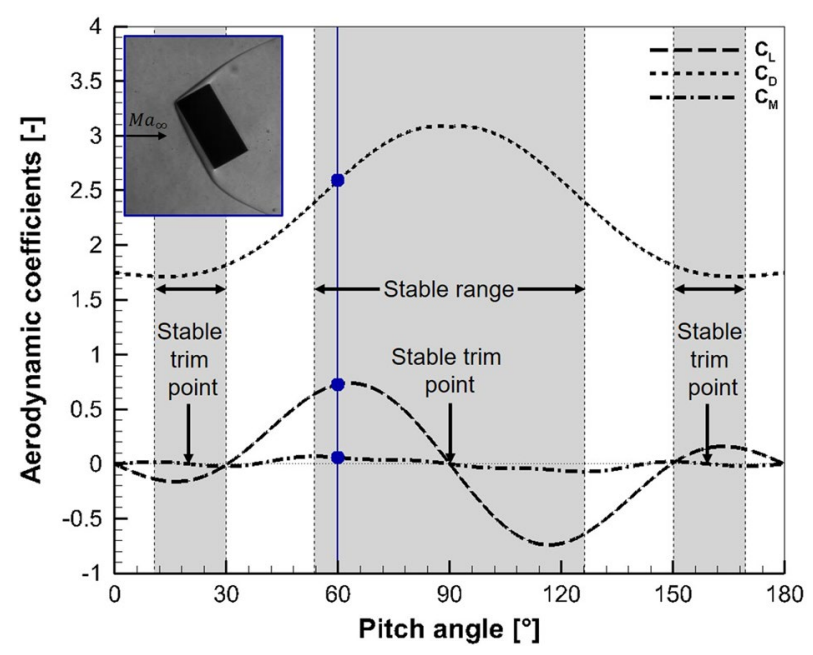

Keywords Atmospheric entry $\cdot$ Hypersonic wind tunnel $\cdot$ High-speed aerodynamics $\cdot$ Free-flight technique $\cdot$ CFD simulation $\cdot$ Shock-shock interaction

Patrick M. Seltner

patrick.seltner@dlr.de

Extended author information available on the last page of the article 


\section{Introduction}

Aerodynamics of simple-shaped bodies in high-speed flows is generating renewed interest in the field of atmospheric entry of meteoroids, space debris and separating components of launch systems. Especially in the past decade, the characterization of flowfields around single (Lee et al. 2017; Seltner et al. 2019; Rees et al. 2020; Grossir et al. 2020) and multiple bodies (Laurence et al. 2012; Marwege et al. 2018; Park and Park 2020; Register et al. 2020) has gained importance with respect to fragmentation, demise and separation behaviors of these space objects undergoing atmospheric entry. The determination of their flight trajectories helps to predict the impact area of uncontrolled entering spacecrafts with the objective to protect persons and properties from harm. The flight attitude of non-spherical bodies has an effect on the flowfield around the object as well as the resulting aerodynamic and thermal loads (Seltner et al. 2019). Yet, state-of-the-art engineering tools for re/entry analysis are still not accurate enough to satisfactorily model this effect.

In the first half of last century, development of both high-velocity projectiles and missiles motivated studies of cylindrical objects with both blunt and streamlined ends. The investigations sought to characterize aerodynamic behavior over a wide range of Mach numbers (Vennard 1940; Rouse 1946; Gowen and Perkins 1953; Hoerner 1965). Vennard (1940) and Rouse (1946) called attention that drag coefficient $C_{D}$ significantly depends on Mach number as well as it is affected by the forebody shape in axial flows at transonic and supersonic speeds. Moreover, they suggested to decrease drag by streamlining the body. The extent of drag reduction here hinges on the nose-to-length ratio of cylinder's face, which was found by Hoerner (1965) with the help of his extensive collection of experimental data. Gowen and Perkins (1953) have shown in their analysis by circumferential surface pressure measurements that the Reynolds number in supersonic flow regime reveals no appreciable effect on surface pressure and drag coefficient of cylinders in crossflows and a Reynolds number range from $10^{5}$ to $10^{6}$. Wind tunnel tests with oblique cylinders at Mach number 6.86 and angle of attack (AoA) between $15^{\circ}$ and $90^{\circ}$ were carried out by Penland (1954), implying a dependency of the angle of attack on lift and drag coefficient. Furthermore, they identified an almost constancy of the drag coefficient for Mach numbers higher than four for cylinders in crossflow with an approximate value of $C_{D}=1.24$ (based on the reference area $S_{\text {ref }}=l d$ ). The diameter and length of cylindrical body are denoted as $d$ and $l$, respectively. In contrast, Penland, Hoerner's value tends to a constant level of $C_{D}=1.21$ for hypersonic inflows based on the same experimental dataset in combination with additional datasets at similar test conditions. For a cylinder in axial flow, he stated a rough convergent value of $C_{D}=1.65$ (based on $S_{\text {ref }}=\pi d^{2} / 4$ ), while the base drag of both configurations tends toward zero at hypersonic speeds. Furthermore, Hoerner mentioned that the cylinder length has no noticeable effect on drag coefficient under supersonic axial-stream as tested in the aspect ratio $(l / d)$ range from 2 to 5 . Maslach and Schaaf (1962) investigated the drag of cylinders in the transition from continuum to free molecular flow under supersonic conditions, which suggests a continuous increase between the two flow regimes.

Based on the short review presented above, previous research has tended to focus merely on drag of cylindrical bodies in axial and crossflows at supersonic speed. The issue of inclined right circular cylinders in hypersonic flows, however, has not been studied so far. Moreover, aerodynamic moment components involved due to the AoA has not been determined. The main weakness in previous studies is the experimental setup. Interferences occur in the setups of (Gowen and Perkins 1953; Hoerner 1965; Maslach and Schaaf 1962), whereby the walls of wind tunnel affect a small disturbance in the flow. Gowen and Perkins 's and Penland 's approach of drag coefficient determination with the help of circumferential pressure distribution on circular cylinders is limited on a two-dimensional measurement by use of their applied setups that do not take the aerodynamic effects of the body ends into account. As a result of previous analysis, several authors determined a drag coefficient for similar test conditions, which exhibits a variance in the literature for cylinders in crossflows.

The present paper aims to address this gap by offering results of experimental and numerical investigations of pitched cylindrical bodies in hypersonic flow, whereby the aerodynamic drag force, lift force and pitching moment are ascertained. Therefore, experiments were performed in the hypersonic wind tunnel Cologne (H2K) of the DLR applying a free-flight technique in combination with non-contact stereo-tracking measurement technique based on a stateof-the-art digital image correlation (DIC) algorithm. These kinds of methods are well-established and commonly used measurement technique as in Preci et al. (2015), Beberniss and Ehrhardt (2017), Riley et al. (2019), Daub et al. (2020a, 2020b). For numerical computations, NASA's inviscid flow solver Cart3D according to Aftosmis et al. $(1998,2000)$ as well as compressible Navier-Stokes solver US3D according to Nompelis et al. (2005) was used.

This article begins by describing experimental and computational tools as well as the setup, followed by presenting and discussing the results. In addition, Sect. 3 contains a comparison between the aerodynamic coefficients of the wind tunnel tests and the two computational fluid dynamics (CFD) tools. 


\section{Methods and models}

\subsection{General setup}

Figure 1 shows the two right circular cylindrical bodies that serve as models for this investigation having a diameter of $0.05 \mathrm{~m}$ and a length of $0.1 \mathrm{~m}$. The solid line shows a simple constant radius cylinder, while the dashed line indicates the concave or "dogbone" model. This body shape is a generic geometry inspired by rocket engines that belong to the rocket-mission-related objects of space debris representing a significant amount of reentered objects in 2020 (ESA Space Debris Office 2020). An axially symmetrical cavity with a parabolic shape is defined for the dogbone, whereby its local radius $r$ depending on the axial coordinate $x$ is given in Eq. (1).

$r(x)= \begin{cases}\frac{\frac{\mathrm{d}}{2}-\frac{\mathrm{d}_{c}}{2}}{\left(\frac{l_{c}}{2}\right)^{2}} \cdot x^{2}+\frac{\mathrm{d}_{c}}{2} ; & |x|<\frac{l_{c}}{2} \\ \frac{\mathrm{d}}{2} & ; \quad \frac{l_{c}}{2} \leq|x| \leq \frac{l}{2}\end{cases}$

This geometry features a minimum diameter $d_{c}$ of $0.02 \mathrm{~m}$ and a cavity length $l_{c}$ of $0.06 \mathrm{~m}$. For the experimental part, the models of both geometries were manufactured of structural steel with the standardized material number 1.0037 . The mass was measured with a precision scale, and the moment of inertia was computated based on the actual mass and length. In doing so, the test articles with cylinder geometry have a mass of $1.54 \mathrm{~kg}$ and a transverse inertia of $1.53 \cdot 10^{-3} \mathrm{~kg} \mathrm{~m}^{2}$, while the test objects with dogbone geometry exhibit a mass of $0.97 \mathrm{~kg}$ and a transverse inertia of $1.30 \cdot 10^{-3} \mathrm{~kg} \mathrm{~m}^{2}$.

Figure 2 depicts the reference frame of the entirely freemoving object, where $x, y$ and $z$ are the Cartesian coordinates as well as $g$ is the gravitational acceleration. The definition of the reference frame is needed to determine its aerodynamic coefficients of lift force $C_{L}$, drag force $C_{D}$ and pitching moment $C_{M}$. The origin of the right-handed global coordinate system (GCS) here is spatially fixed in the center of nozzle's exit plane. It has the positive $x$-axis

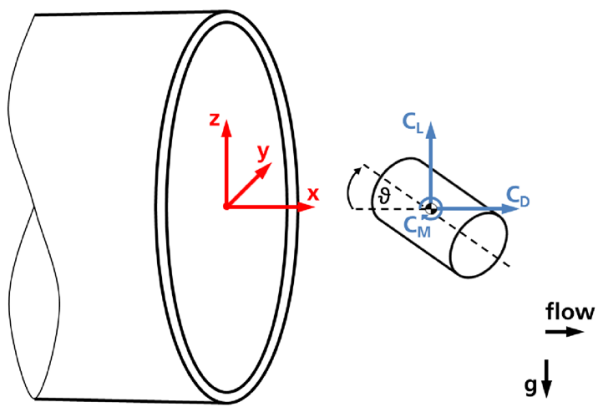

Fig. 2 Global coordinate system with respect to nozzle exit and definitions of the pitch angle $\vartheta$ and the aerodynamic coefficients $C_{D}, C_{L}$ and $C_{M}$

pointing downstream, the $y$-axis in the transverse direction and the positive $z$-axis pointing vertically up. A body-fixed local coordinate system (LCS) is defined in the center of gravity $(\mathrm{CoG})$ of the free-flight object that $x$-axis is congruent with the symmetry axis of the cylinder. Unlike, the aerodynamic coefficients of this object are specified in the spatially fixed reference frame of the GCS. The influence factor of interest in this study is the pitch angle $\vartheta$ (shown in Fig. 2), which designates the inclination between the global $x$-axis and cylinder's symmetry axis. The positive direction of rotation is counter-clockwise when viewed to the $y$-axis. Hence, $\vartheta=0^{\circ}$ means that the base area is exposed to the flow. This configuration is called base-exposed in the following. A further appearing orientation designation in this article is side-exposed, whereby the symmetry axis of the cylindrical body is orthogonal to the flow direction.

For the flow conditions (FC) used in this study, the main parameters are listed in Table 1, whereby the numerical investigations were performed with a theoretical reservoir pressure of 5.2 bar and the wind tunnel tests were run at 4.8 bar.

\subsection{Experimental tools}

The experimental setup and test procedure were largely similar to that in previous work with free-flying cubes Seltner et al. (2019). The following section briefly describes
Fig. 1 Dimensioned geometry of cylinder (solid line) and dogbone (dashed line) including axis of revolution

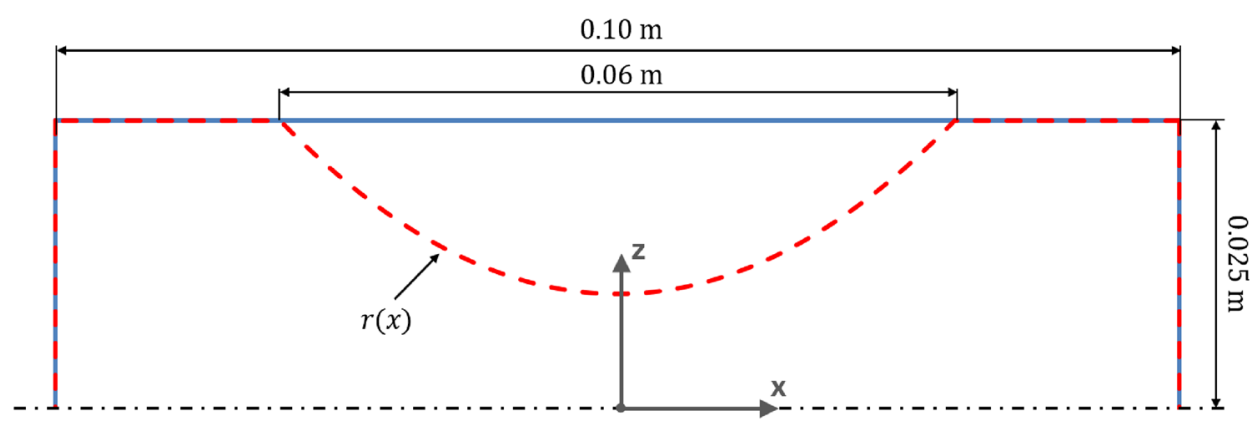


Table 1 Typical flow conditions

\begin{tabular}{lll}
\hline Quantity & Numerical FC & Experimental FC \\
\hline $\begin{array}{l}\text { Free-stream Mach number } M a_{\infty} \\
\quad[-]\end{array}$ & 7.0 & 7.0 \\
Reservoir pressure $p_{0}[\mathrm{bar}]$ & 5.2 & 4.8 \\
Reservoir temperature $T_{0}[\mathrm{~K}]$ & 600 & 600 \\
Free-stream static pressure $p_{\infty}[\mathrm{Pa}]$ & 126 & 116 \\
Free-stream temperature $T_{\infty}[\mathrm{K}]$ & 56 & 56 \\
Free-stream density $\rho_{\infty}\left[\mathrm{g} / \mathrm{m}^{3}\right]$ & 7.8 & 7.3 \\
Free-stream velocity $v_{\infty}[\mathrm{m} / \mathrm{s}]$ & 1046 & 1046 \\
Free-stream unit Reynolds number & 2.1 & 2.0 \\
$R e_{\infty}\left[10^{6} / \mathrm{m}\right]$ & & \\
\hline
\end{tabular}

the main details, minor modifications as well as the model release mechanism, measurement technique and post-processing system.

\subsubsection{Hypersonic wind tunnel Cologne (H2K)}

The experiments were conducted in the hypersonic wind tunnel Cologne H2K located at the Supersonic and Hypersonic Technologies Department of the DLR. This wind tunnel is an intermittently working blowdown facility with axisymmetric contoured Laval nozzles for fixed design Mach numbers and a free-jet test section. The flow at nozzle exit has a fully developed turbulent boundary layer. To avoid condensation of air during expansion in the nozzle, the facility is equipped with eight electrical heaters with a maximum power of $5 \mathrm{MW}$. By varying the reservoir pressure $p_{0}$ and the reservoir temperature $T_{0}$, the unit Reynolds numbers can be adjusted. The major facility attributes of $\mathrm{H} 2 \mathrm{~K}$ are mentioned in Table 2.

For the present tests, a Mach-7 nozzle was employed, which emits a flow with turbulent boundary layer into the vacuum of the test section. A free jet with an axially symmetrical homogenous flow and a shear layer between core flow and ambient vacuum develops. Fig. 3 illustrates its flow characteristics from experimental measurement for the applied nozzle as the ratio of locally measured and theoretical ram pressure, whereby the measured quantity $p_{r}$ was

Table 2 H2K's facility attributes

\begin{tabular}{ll}
\hline Parameter & Feasible values \\
\hline Mach number & $5.3,6.0,7.0,8.7,11.2$ \\
Unit Reynolds number & $2.0-20 \cdot 10^{6} / \mathrm{m}$ \\
Nozzle exit diameter & $600 \mathrm{~mm}$ \\
Reservoir pressure & $2.5-55 \mathrm{bar}$ \\
Reservoir temperature & $\max .1000 \mathrm{~K}$ \\
Typical run time & $30 \mathrm{~s}$ \\
\hline
\end{tabular}

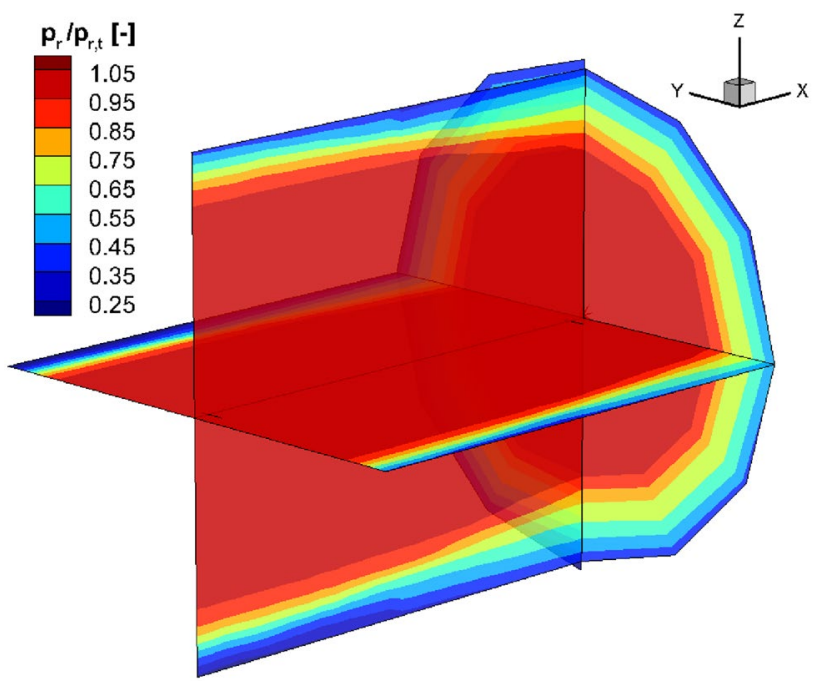

Fig. 3 Ratio of locally measured ram pressure to theoretical ram pressure of the free jet for the Mach-7 nozzle (flow conditions: $p_{0}=23.5$ bar, $T_{0}=662 \mathrm{~K}, \operatorname{Re}_{\infty}=8.3 \cdot 10^{6} / \mathrm{m}$ ); flow direction is the positive $\mathrm{x}$-direction; core flow is defined by the red area

determined with pitot probes and the theoretical quantity $p_{r, t}$ is defined as the adiabatic stagnation pressure behind a normal shock based on the reservoir pressure. Here, the maximum deviation is $5 \%$ in the core flow. However, the calibration measurements were carried out with a higher Reynolds number $\left(\operatorname{Re}_{\infty}=8.3 \cdot 10^{6} / \mathrm{m}\right)$ than for the freeflight tests. Thus, the present core flow size is a bit smaller, in fact. A detailed description of nozzle calibration is given in Niezgodka (2001).

\subsubsection{Free-flight technique}

A free-flight technique was applied for the present tests as explained in Seltner et al. (2019) allowing an entirely free movement of the model in flow. By the use of this technique, aerodynamic interferences due to a sting support or a balance are prevented. In the beginning, the measurement chamber is in vacuum condition and the test article is held by an electromagnet located at the ceiling of the chamber. The model alignment on the holder defines the initial pitch angle $\vartheta_{0}$. To adjust this angle, an adjustable angle mounting plate is used to cover a broad range of pitch angle. When the free jet is stable, the test model is detached from the holder and falls in vacuum. By passing the shear layer, the increasing stagnation pressure (shown in Fig. 3) causes a rotation of the body. Subsequently, the effective test time of roughly $0.1 \mathrm{~s}$ begins as soon as the model is completely within the homogenous core flow. At the end, the object crosses the lower part of the shear layer before it is caught at the bottom of the test section. For the present experimental setup, two or three 
model releases per run of the wind tunnel were realized to reduce the operational costs, whereby a time delay between the drops is satisfied to ensure a steady state of the free jet for each free-flight.

\subsubsection{Instrumentation}

The facility operation conditions $p_{0}$ and $T_{0}$ were acquired with a sampling rate of $20 \mathrm{~Hz}$. GE Druck's absolute pressure transducer of the UNIK 5000 series with a total accuracy of $\pm 2.8 \mathrm{kPa}$ was in use for the reservoir pressure measurement. It ensures a 2.5 times higher precision than the utilized sensor of previous tests in Seltner et al. (2019). For the reservoir temperature, a thermocouple type K class 1 from MTB Sensor-Technik was applied having a total accuracy of $\pm 1.5 \mathrm{~K}$. In addition, a systematic error increases the uncertainty of $T_{0}$ by $\pm 4.0 \mathrm{~K}$. Due to the response time of the thermocouple, the test time is too short for the sensor to reach equilibrium at the current test conditions. Thus, an extrapolation of $T_{0}$ was carried out to determine the actual reservoir temperature.

High-speed schlieren photography was used to characterize the flowfield around the test article. To capture sharp images, the optical schlieren setup was arranged as Z-type system as described in Seltner et al. (2019). This system was equipped with a $1000 \mathrm{~W}$ xenon arc lamp as a light source and a Photron FASTCAM APXRS model $250 \mathrm{~K}$ high-speed camera for image recording. The camera was set to a frame rate of $3 \mathrm{kHz}$ at full $1024 \times 1024$ pixels resolution and an exposure time of $4 \mu \mathrm{s}$.

To reconstruct the three-dimensional flight trajectory and attitude of the freely flying model, a high-speed stereo-tracking system based on the detection of marker points on the model surface was applied. Two synchronously recording cameras in stereoscopic arrangement were placed inside the test section above one schlieren window having a view on the measurement volume at an angle of roughly $60^{\circ}$ against the horizontal plane in order not to conceal the schlieren path. Both cameras were from the same model: Photron FASTCAM SA-X2 model $480 \mathrm{~K}$. They operated with a full-frame resolution of $1024 \times 1024$ pixels at a frame rate of $12 \mathrm{kHz}$. With 24-mm-focal-length lenses and a measurement distance of roughly $700 \mathrm{~mm}$, a measurement volume of $590 \times 625 \times 625 \mathrm{~mm}$ is observed for the present tests. These two cameras were enveloped in sealed boxes to prevent overheating due to the surrounding vacuum during the operation of wind tunnel. Each camera was equipped with four $86 \mathrm{~W}$ plus four $38 \mathrm{~W}$ high-power LEDs on a capacitive cooling ring, which was concentrically mounted on the protective box for optimal illumination.

\subsubsection{Data processing}

The recorded sequence of synchronous image pairs from the stereo-tracking cameras is used to determine the center-ofgravity position in 3D space as well as body orientation of the model motion. A point-based DIC technique is applied therefore by the use of the commercial stereo-tracking software ARAMIS Professional 2017 providing threedimensional and time-resolved data about coordinates and displacements of a point or a group of points in a measurement volume by the principle of triangulation. This software allows a $6 \mathrm{DoF}$ analysis of deformations and motions in general. For the present investigation, the software is used to determine the three positions in streamwise $(x)$, spanwise $(y)$ and vertical $(z)$ direction as well as the orientations roll $(\varphi)$, pitch $(\vartheta)$ and yaw angle $(\psi)$ of the test object with respect to the GCS.

With the time-resolved motion data, the velocities and accelerations of the test object are calculated by filtering thrice and differentiating twice. The discrete differentiation is performed by central finite difference quotient. Because of the low signal-to-noise ratio in the data of the motion derivatives, a conventional Savitzky-Golay filter is utilized for digital smoothing, which fits a polynomial of degree 1 to segments of 201 (cylinder) or, respectively, 121 (dogbone) adjacent data points for the present setting. This particular filter was chosen to remove higher frequency components without completely cutting off them from the signal, whereby this configuration promises the best results after a careful analysis of parameters.

For the calculation of the aerodynamic coefficients, the test time was chosen in such a way that the free jet is stabilized and the model is entirely within the homogenous core flow (red area in Fig. 3) to minimize acceleration uncertainties due to the stagnation pressure deviation. The force and moment coefficients are calculated with the formula as in Seltner et al. (2019), where the reference area is the base area of the cylinder $\left(S_{\text {ref }}=\pi \mathrm{d}^{2} / 4\right)$, and the reference length is the cylinder's diameter. To determine the lift coefficient, the vertical ( $z$ ) force component is separated from gravity, assuming a gravitational acceleration of $g=9.81127 \mathrm{~m} / \mathrm{s}^{2}$ according to Achner (2011). More details can be found in a previous publication (see Seltner et al. 2019).

In addition, a curve fitting by the method of least absolute residual is applied to the motion derivatives of the measured data to establish a quantitative relationship between aerodynamic coefficients and pitch angle. Based on the deviations between measured and fitted data, the experimental uncertainties of the aerodynamic coefficients are determined by a simple error estimation in the following analysis. For this purpose, the statistical error of regression in a 3- $\sigma$ neighborhood is used, where $\sigma$ is the standard deviation. Furthermore, the impact of the smoothing is considered for the overall 
uncertainty. A trajectory, determined by the aerodynamic coefficients of the curve fitting, is processed therefore with the present setting of filtering and differentiating to obtain coefficients, which are compared with its original one.

Analytically determined aerodynamic coefficients under hypersonic conditions are calculated for comparison reasons by the use of the simple modification of the Newtonian flow theory as described in Lees (2003). This hypersonic approximation is applied to a three-dimensional body, where the windward surface elements are impinged with pressure coefficients determined by the Newtonian squared sine term, while the leeside elements experience a zero pressure coefficient.

\subsection{Computational tools}

Two flow solvers commonly used by NASA were applied in the present work containing the inviscid Cart3D code (see Sect. 2.3.1) and the viscous US3D code (see Sect. 2.3.2).

\subsubsection{Cart3D code}

NASA's Cart3D simulation package performs inviscid aerodynamic simulations and is based upon a fully automated Cartesian cut-cell approach. The Euler equations are discretized on a multilevel Cartesian mesh with embedded boundaries according to Aftosmis et al. $(1998,2000)$. These meshes consist of regular Cartesian hexahedra everywhere, except for a layer of body-intersecting cut-cells at solid wall boundaries. While the mesh consists of nested Cartesian cells, it is viewed as an unstructured collection of control volumes making the approach well-suited for parallel computation and solution-adaptive mesh refinement. Steady-state flow solutions are obtained using a five-stage Runge-Kutta scheme with local time stepping and h-p multigrid, which uses coarsening of both the physical mesh and polynomial representation of the solution to accelerate convergence. Domain decomposition via space-filling curves permits parallel computation with excellent scalability. Meshes are automatically generated using adjoint-based adaptation. The duality-preserving discrete adjoint leverages the domain decomposition and other infrastructures from the primal solver and provides both error estimation and output-driven mesh adaptation (Nemec and Aftosmis 2016). For the cases discussed here, each simulation used package's adaptive meshing capability where the adaptation goal was to reduce the discretization error in computing loads on the cylinders. Results published by Register et al. (2020) show excellent agreement with both experiment and unsteady Navier-Stokes simulations for flow over multiple interacting spheres in supersonic flow.

\subsubsection{US3D code}

The US3D solver was chosen for the viscous simulations. US3D is an unstructured finite volume Navier-Stokes solver according to Nompelis et al. (2005). The unstructured formulation allows for a broader range of mesh topologies and element types which can enable simulations of complex geometries. The solver uses a range of spatial and temporal schemes as well as several turbulence models to enable a broad range of flow regimes to be solved. For the flows presented here, a second-order backward difference time integration scheme based on the data-parallel-line-relaxation (DPLR) (see Wright et al. 1998) and full matrix data parallel (FMDP) (see Nompelis et al. 2005) implicit method is used. Spatial fluxes are computed using a sixth-order low-dissipation inviscid flux scheme by Subbareddy and Candler (2009) and second-order viscous fluxes. The low dissipation fluxes allow for better resolution of strong gradients and smaller flow features on a given mesh resolution compared to upwind methods. Finally, turbulence modeling closure was achieved using the one-equation turbulent viscosity eddy model according to Spalart and Allmaras (1992) that is cast in a hybrid of Reynolds-averaged Navier-Stokes (RANS) and large eddy simulation (LES) formulation known as the detached eddy simulation (DES97).

\section{Results}

The aim of the following analysis is to further current knowledge of cylinder aerodynamics in hypersonic flowfields with main focus on the effect of body inclination on flow structures influencing the single-body aerodynamic forces and moments. For this propose, the $\operatorname{drag}\left(C_{D}\right)$ and lift $\left(C_{L}\right)$ forces as well as the pitching moment $\left(C_{M}\right)$ relating to the pitch angle $(\vartheta)$ are investigated. Therefore, new-found experimental and computational results of wind tunnel tests and CFD simulations, respectively, are depicted and compared with each other. This section begins by illustrating the flow characteristics in Sect. 3.1 followed by presenting the reconstructed model motion of the free-flight tests in Sect. 3.2. The next two subsections looks at the motion derivatives after post-processing enabling the determination of cylinder's statically stable flight attitudes. Finally, the influence of an axially symmetrical cavity is examined in the last subsections.

\subsection{Experimental and computational flow visualization}

The primarily examined parameter in this research is the pitch angle $\vartheta$. A value of $\vartheta=0^{\circ}$ here signifies that the base of cylinder is orthogonal to the inflow as described 


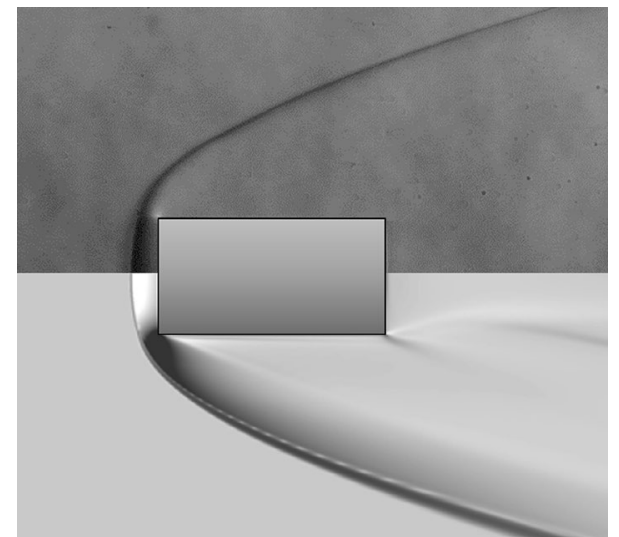

Fig. 4 Comparison of experimental (top) and computational (bottom) flowfield around a cylinder at $\vartheta=0^{\circ}$

in Sect. 2.1. A visualization of the density gradients in $\mathrm{x}$-direction of this base-exposed attitude under the equal flow conditions is depicted in Fig. 4 presenting the flow structures around the body. The upper part of Fig. 4 shows the integrated density gradient through the fluid domain in the schlieren image from free-flight tests, whereas the lower part depicts the computational image of the gradient merely on the central plane coming from US3D simulations. This qualitative comparison of the experimental and computational flowfields shows a very good agreement, whereby the bow shock shape and stand-off distance seem to be identical. However, an expansion fan on the trailing edge followed by flow separation as well as shocks at the body's shell surface downstream the leading edge and also at the shear layer of the wake region is presented just in the computational image, which are not visible on the schlieren image due to the low sensitivity of the schlieren system to free-stream density.

A sketch of the supersonic flow phenomena with the corresponding experimentally determined schlieren photograph in the background is shown in Fig. 5, which is consistent with the flow topology of blunt bodies as in the established literature (Hoerner 1965) as well as in a previous study of the authors with a cube (Seltner et al. 2019). Most notable here is the occurrence of a weak reattachment shock as a result of a tiny separation bubble immediately downstream the leading edge. This type of separation is called shoulder separation as described in Kaufman II et al. (1966), which is caused by an adverse pressure gradient because of recompressions of the flow after a strong expansion. Such a separation on cylinder's shell surface was also observed in Matthews and Eaves Jr. (1967).

For a quantitative analysis of the gas dynamic flow structures, CFD simulations were performed using US3D considering viscous effects. Figure 6 illustrates the flowfield around a base-exposed cylinder with isothermal boundary condition of their walls. In Fig. 6a, the Mach number distribution with

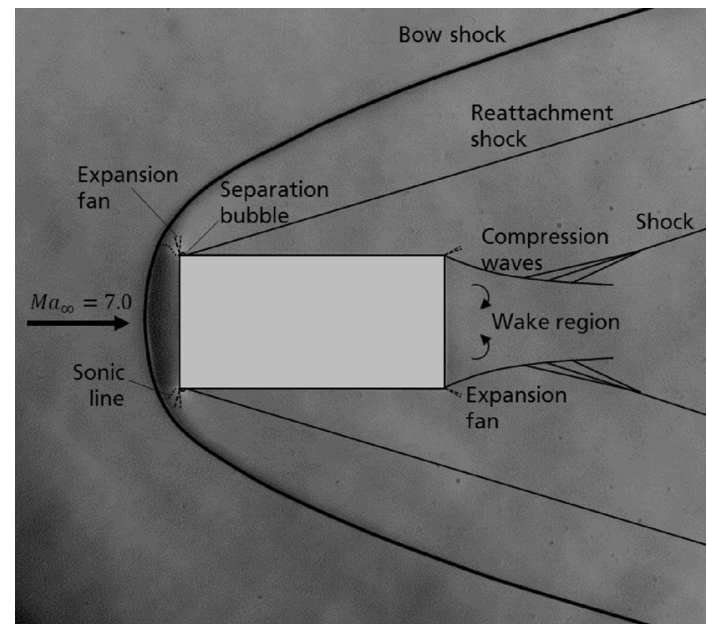

Fig. 5 Qualitative flowfield of one cylinder's base is exposed to the flow

streamlines is shown. This color contour plot confirms the observations from schlieren images that the Mach number falls below one in front of the windward surface. Downstream of both body edges, a rise of the Mach number is apparently being caused by expansion fans, whereby an increase in Mach number due to the previously mentioned recompression is visible subsequent to the expansion on the leading edge. The recirculation zone in the wake region of the body has a length equal to the cylinder's diameter, whereby this part is subsonic. In Fig. 6b, the distribution of the flowfield temperature as well as the surface pressure is depicted. The bow shock here effects a sudden increase in the flow temperature due to the compression, whereby the highest values with over $550 \mathrm{~K}$ are reached in the subsonic region between bow shock and cylinder's front face. Furthermore, a second region of high-temperature occurs within the wake region having temperatures up to $400 \mathrm{~K}$. Interestingly, the highest values are reached in the supersonic part instead of the subsonic part. Also, a thin boundary layer with higher flow temperatures than in the free stream is visible in Fig. 6b around cylinder's shell. The surface pressure immediately decreases downstream the leading edge, rises afterward until a third of body's length and finally decreases until the trailing edge. Thus, an adverse pressure gradient occurs in the first third of cylinder's shell surface provoking a boundary layer separation as shown in the schlieren images (see Fig. 4).

As soon as the cylinder is inclined, the flowfield around the body becomes asymmetric as well as aerodynamic phenomena become more complex, which is illustrated by means of US3D's numerical schlieren images in Fig. 7. All of these flow structures contain detached bow shocks, whereas the shock shape and stand-off distance depend on the flow angle relatively to the body. The more a body edge 

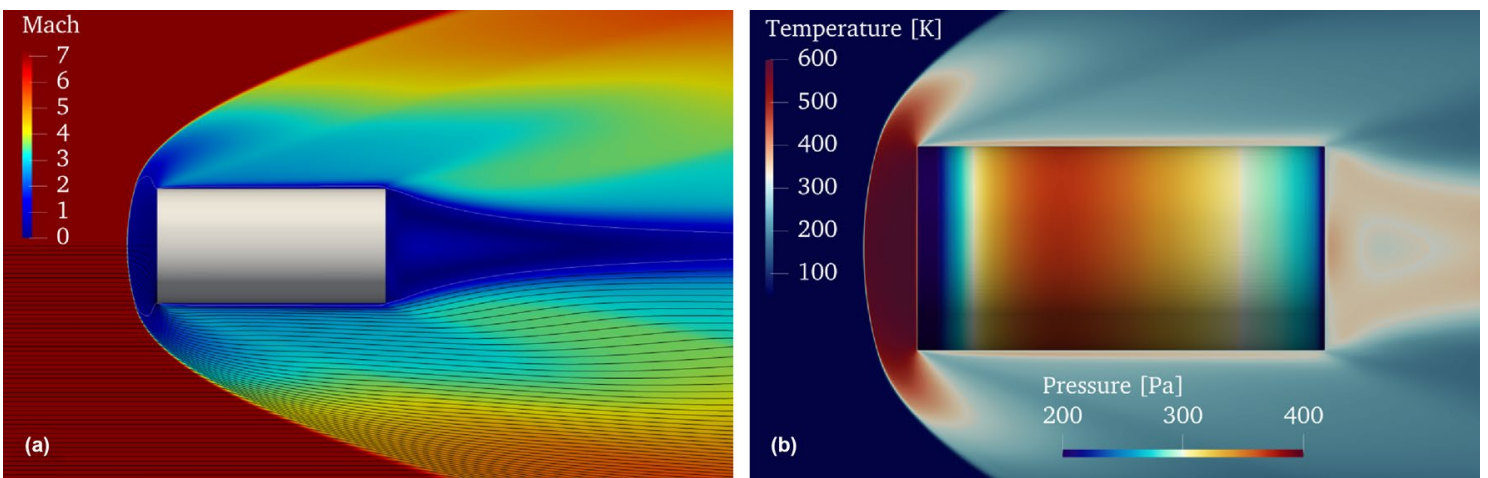

Fig. 6 Computational flowfield of one cylinder's base is exposed to the flow showing distributions of (a) Mach number with streamlines as well as (b) flow temperature with surface pressure

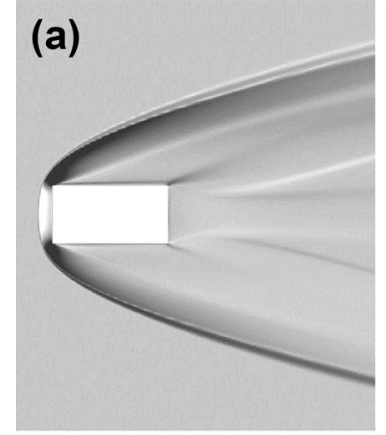

(e)

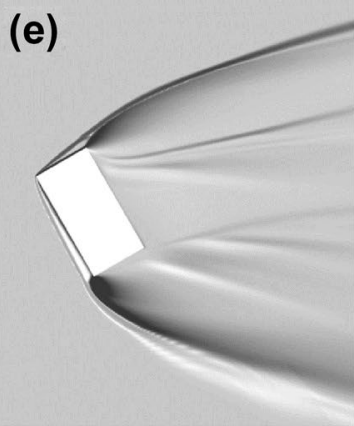

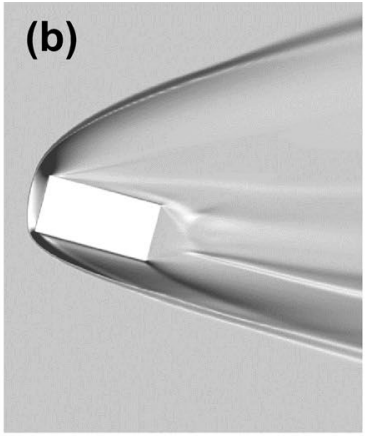

(f)

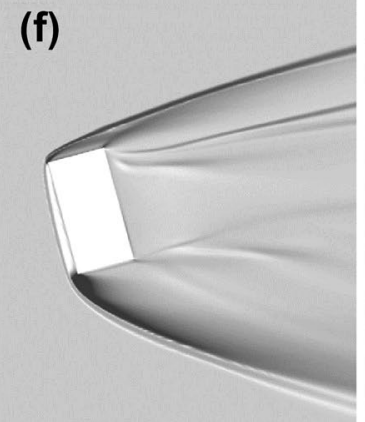

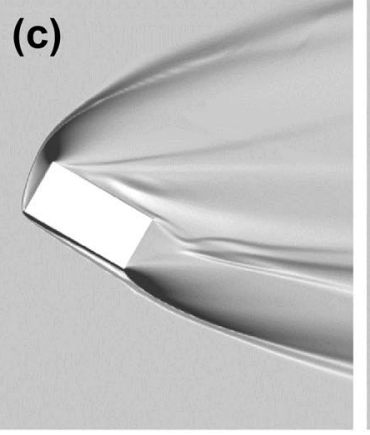
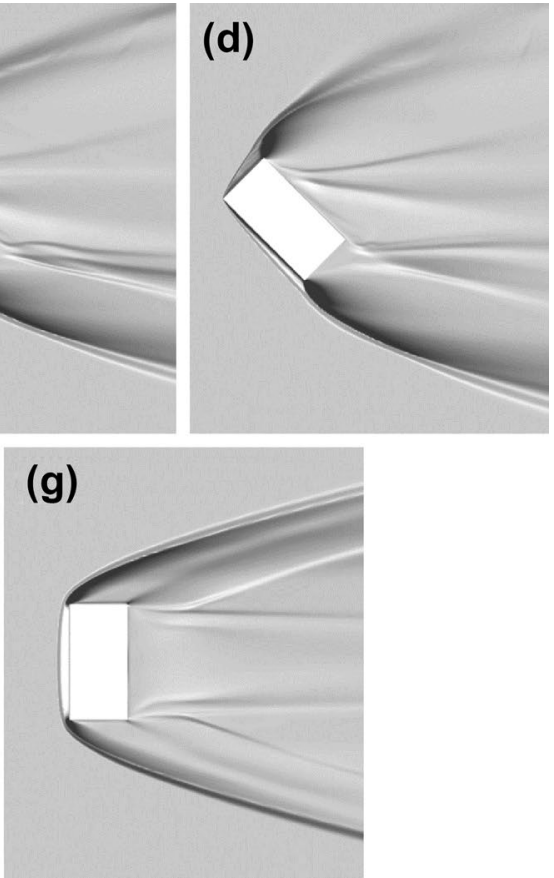

Fig. 7 Pseudo-schlieren images of a cylinder at various pitch angles: (a) $\vartheta=0^{\circ}$, (b) $\vartheta=15^{\circ}$, (c) $\vartheta=30^{\circ}$, (d) $\vartheta=45^{\circ},\left(\right.$ e) $\vartheta=60^{\circ}$, (f) $\vartheta=75^{\circ}$, (g) $\vartheta=90^{\circ}$

protrudes into the flow, the more the shock stand-off distance is reduced, and the shock curvature is greater in the surrounding of the edge. At the leading edges, expansion fans rise that encounter with the bow shock resulting in an aerodynamic interaction associated with a bending of the shock wave over the length of the interaction. In Fig. 7d, a smearing out of the bow shock further downstream is just visible in the numerical schlieren image, which is an artifact due to the reduction of mesh refinement away from the object. Moreover, it seems here that two separate wake regions appear, whereby the flow attaches after the shoulder separation on the leeward shell surface before it detaches again on the furthest edge. Experimental schlieren images from present free-flight tests are presented in Fig. 8. Qualitatively, the stand-off distances and shapes of the bow shocks show a good agreement, and the experimental flowfield is accurately reproduced by the numerical computation (see image of a superposed flowfield as an example in the online supplementary Information).

Furthermore, Fig. 8 qualitatively depicts the motion behavior of several drops with different initial pitch angles, whereby the duration between the first and last image of each sequence is $60 \mathrm{~ms}$. The cylinders here experience streamwise, vertical and pitching displacements. 
(a)
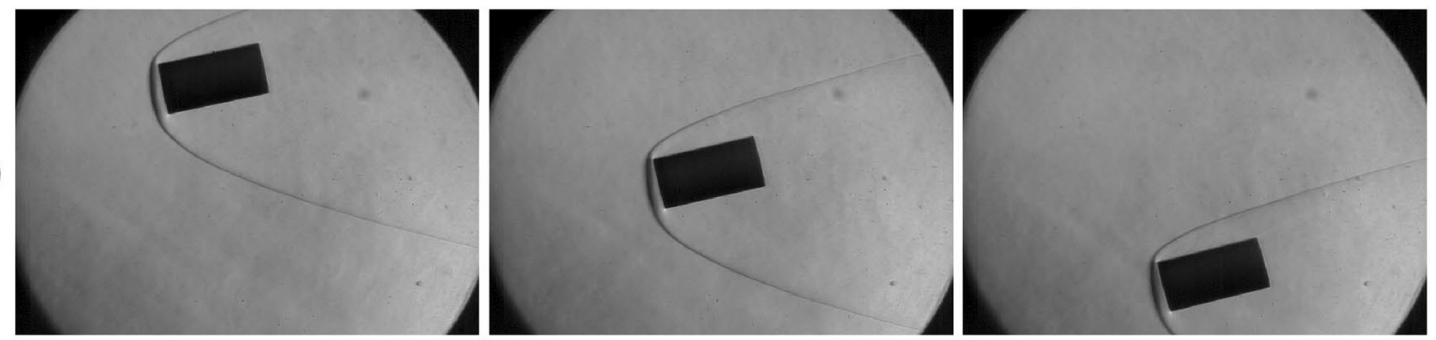

(b)
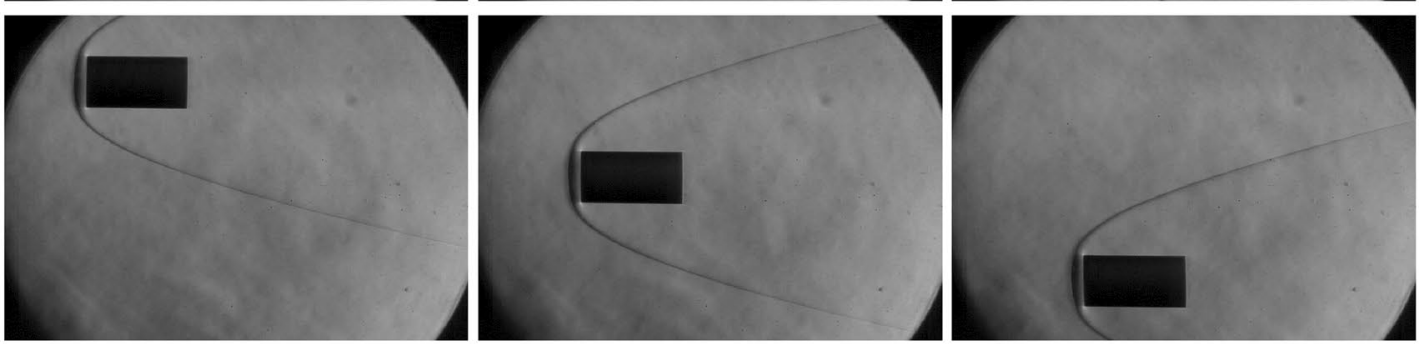

(c)
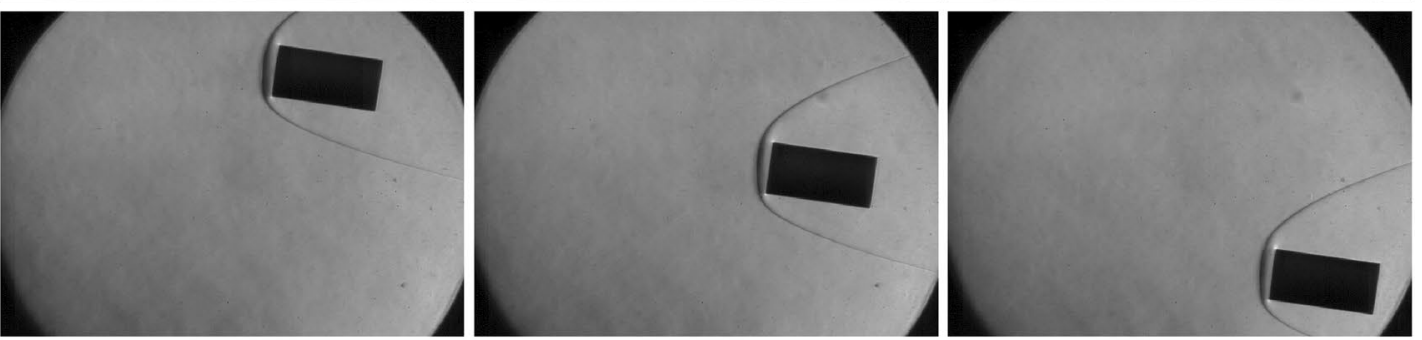

(d)
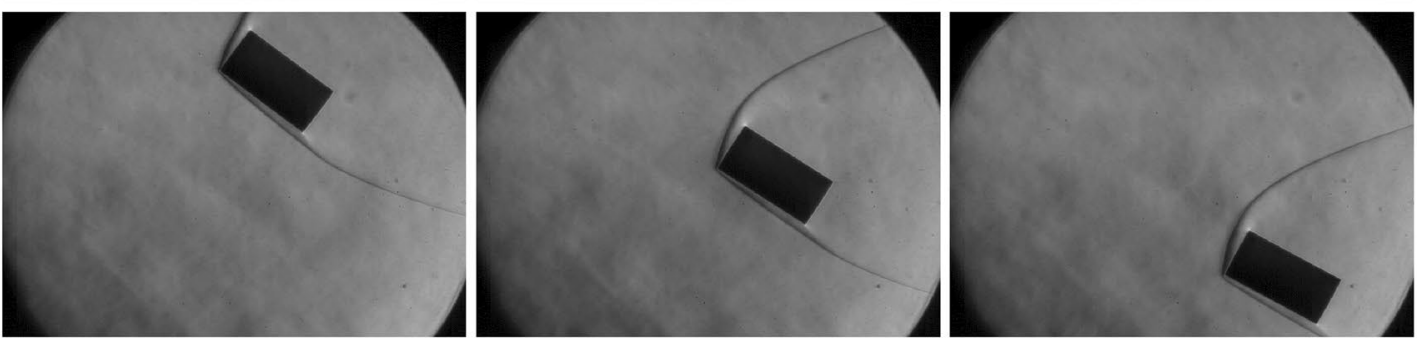

(e)
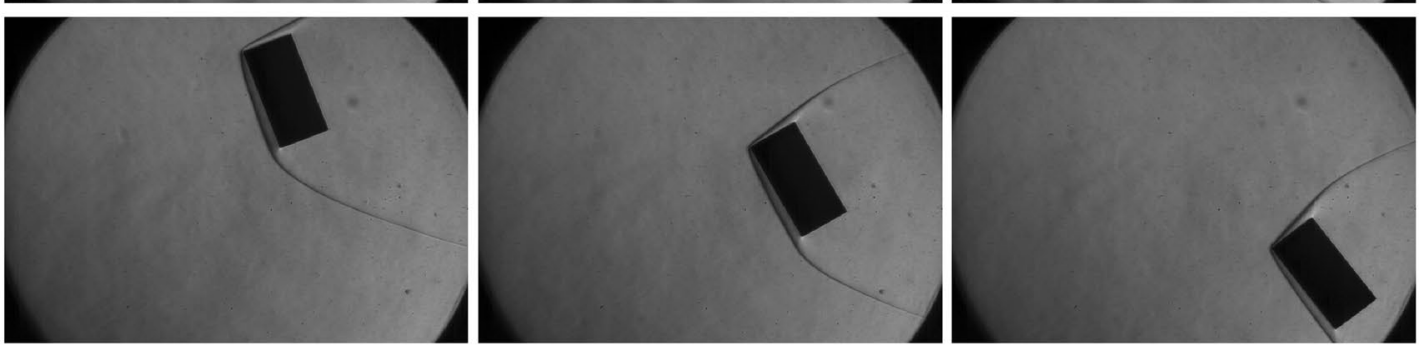

Fig. 8 Selection of schlieren image sequences showing the motion behavior in free-flight with different initial pitch angles: $(\mathbf{a}) \vartheta_{0}=-10^{\circ},(\mathbf{b})$ $\vartheta_{0}=0^{\circ},(\mathbf{c}) \vartheta_{0}=10^{\circ},(\mathbf{d}) \vartheta_{0}=50^{\circ}$ and $(\mathbf{e}) \vartheta_{0}=90^{\circ}$

Regarding the pitching, the bodies rotate counter-clockwise except for $\vartheta_{0}=0^{\circ}$ and $\vartheta_{0}=10^{\circ}$ (shown in Fig. $8 \mathrm{~b}$ and c). The reason for this counter-clockwise rotation is a negative pitching moment caused by an asymmetric surface pressure distribution during the passing through the upper shear layer of the free jet as described in Sect. 2.2.2. For $\vartheta_{0}=0^{\circ}$ and $\vartheta_{0}=10^{\circ}$, the flight attitude does not change, because it seems that a moment, effected by cylinder's flowfield at the current flight attitude, counteracts the rotational motion caused by the free jet's shear layer.

\subsection{Model motion}

By the use of the stereo-tracking system, the motion of the rigid body is reconstructed, whereby each three components of position and orientation are determined. Figure 9 

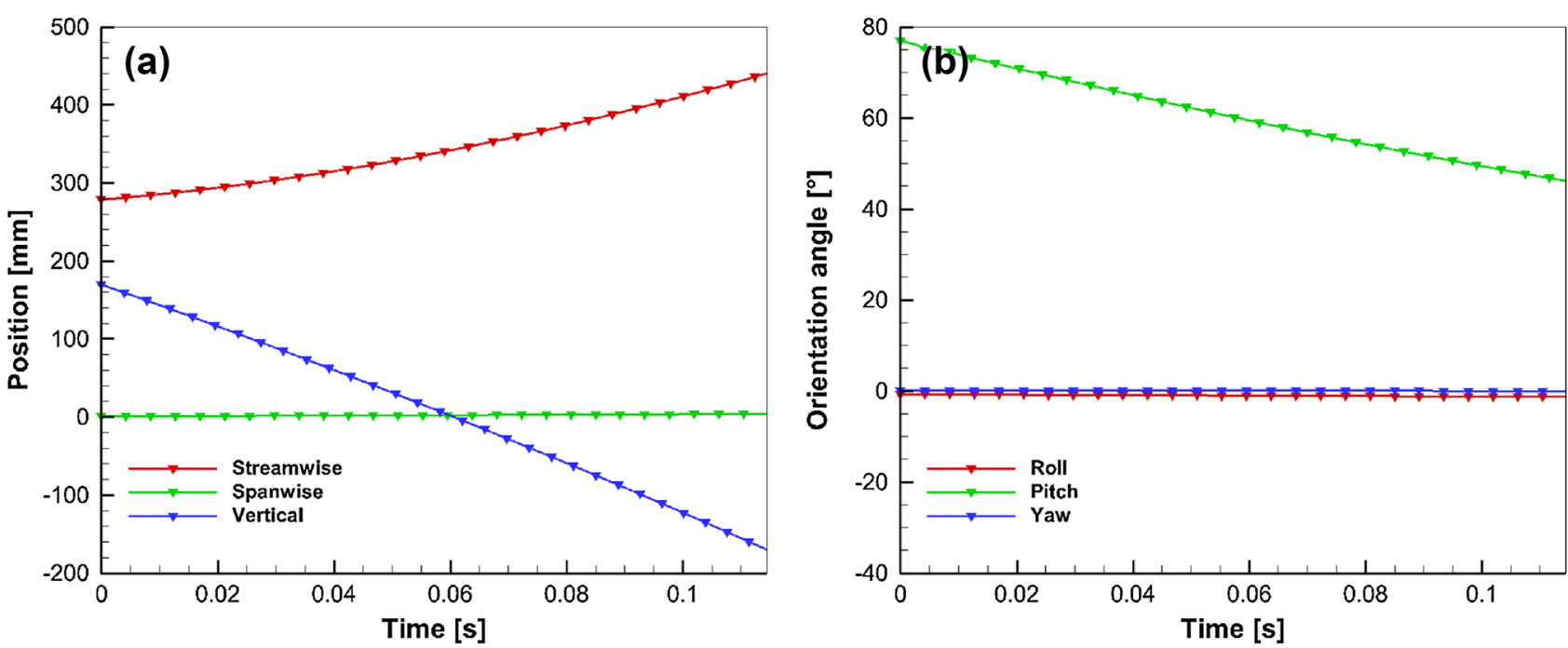

Fig. 9 Evolution of 6 DoF motion displacement in core flow with $\vartheta_{0}=90^{\circ}$ : (a) positions in $x, y$ and $z$ and (b) orientation angles in $\varphi, \vartheta$ and $\psi$

shows an example for the measured displacement of a cylinder configuration with an initial pitch angle of $90^{\circ}$, whereby Fig. 9a depicts the translational displacement (streamwise, spanwise and vertical) and Fig. 9b depicts the angular displacement (roll, pitch and yaw). It is expected that the motion is two-dimensional due to the rotational symmetry of the model as well as the alignment of cylinder's axis and nozzle's axis. This assumption can be confirmed with a view to the displacement data (shown in Fig. 9), whereby the roll, yaw and spanwise motion components are almost zero in contrast to the streamwise, vertical and pitch motion components over the entire freeflight time. Thereby, the changes in streamwise position $(\Delta x=161.3 \mathrm{~mm})$, vertical position $(\Delta z=-339.3 \mathrm{~mm})$ and pitch angle $\left(\Delta \vartheta=-30.6^{\circ}\right)$ are about two orders of magnitude greater than the changes in spanwise position $(\Delta y=3.0 \mathrm{~mm})$, roll angle $\left(\Delta \varphi=-0.6^{\circ}\right)$ and yaw angle $\left(\Delta \psi=-0.2^{\circ}\right)$. Hence, the roll, yaw and spanwise displacements can be neglected.

Figure 10 illustrates the two-dimensional trajectories of all runs in the xz-plane with respect to the center of the nozzle exit plane showing also the ram pressure distribution of the free jet from experimental flow characterizations as a color contour layer. The red area represents the core flow with a homogenous ram pressure distribution. It is apparent that the flight trajectories do not overlap. Unlike the freeflight technique in Seltner et al. (2019), two release mechanisms at two different positions were employed for tests with inclined cylinders, whereby the initial positions in $\mathrm{x}$ - and z-direction vary due to the model alignment. Broadly speaking, a significant streamwise acceleration of all free-flight tests is visible in Fig. 10 as well as the majority of measured data points are within the homogenous flow.

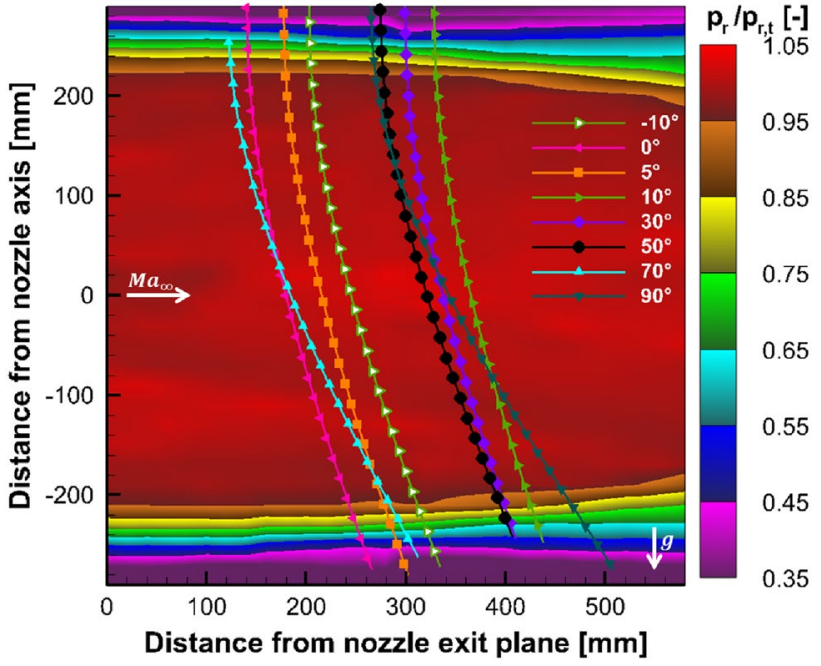

Fig. 10 Flight trajectories of runs with different initial pitch angles and flowfield characteristics of H2K's Mach-7 nozzle

Six-DoF velocities and accelerations of the same exemplary run (as of Fig. 9) are depicted in Fig. 11. As previously reported about the neglect of three motion components, the derivatives of roll, yaw and spanwise motion also confirm the previous observations, because these quantities are roughly zero over time. As a result, the streamwise, vertical and pitching components are only considered for all experimental results presented hereinafter and the motion analysis is $3 \mathrm{DoF}$. The velocities of the streamwise, vertical and pitch motion components in Figs. 11a and 11 b clearly differ from zero at $t=0 \mathrm{~s}$, when the entire body is within core flow. These deviations from zero demonstrate the impact of the free jet's shear layer on the body motion causing a strong 

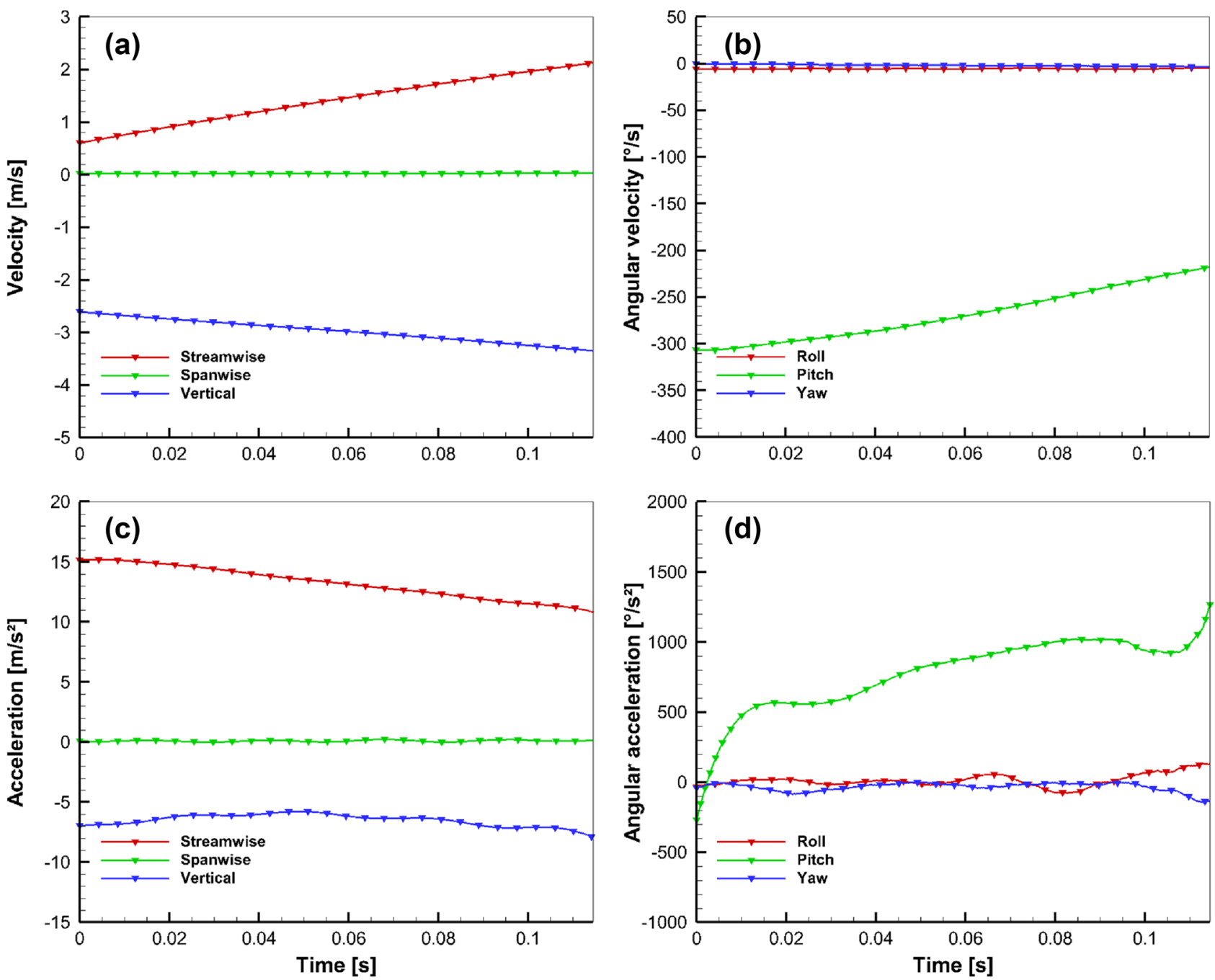

Fig. 11 Evolution of 6DoF translational and angular motion derivatives in core flow with $\vartheta_{0}=90^{\circ}$ : (a) velocities, (b) angular velocities, (c) accelerations and (d) angular accelerations

pitch rotation as well as a significant streamwise acceleration. It is notable that the pitch acceleration in Fig. $11 \mathrm{~d}$ is negative at the beginning $(t=0 \mathrm{~s})$ and turns to positive values shortly after. The reason for this change of sign seems to be that the first values are affected by adjacent data points during the filtering, for which the body experience a negative pitch acceleration due to the shear layer. For the determination of accelerations (second-order derivatives), the data are filtered thrice by use of 100 adjacent data points before and after each value for all filtering steps. Thus, the first and also the last values in Fig. 11d are influenced by measurement points captured within the shear layer, which explains the strong deviations at the edges of the plot and can also be observed for the pitching moment coefficients. In contrast to the angular derivatives, the translational acceleration of cylinders in crossflow seems to be less sensitive to inhomogenous distributions of the ram pressure than the pitch acceleration. Inside the core flow, the pitch acceleration in Fig. 11d has significant positive values, which cause a counter-rotation referred to the motion of the model within the upper shear layer.

\subsection{Aerodynamic forces and moments}

The resulting aerodynamic coefficients derived from the motion derivatives in streamwise (drag force) and vertical (lift force) direction as well as around the spanwise axis (pitching moment) are presented in Fig. 12 as a function of pitch angle. In addition to the experimental data of several runs with different initial pitch angles, trigonometric fitting and hypersonic approximation curves are shown in the diagrams, as well. Aerodynamic coefficients based on two different reference areas are presented here. On the one hand, a constant circular base area (Fig. 12a) and, on the other hands, 

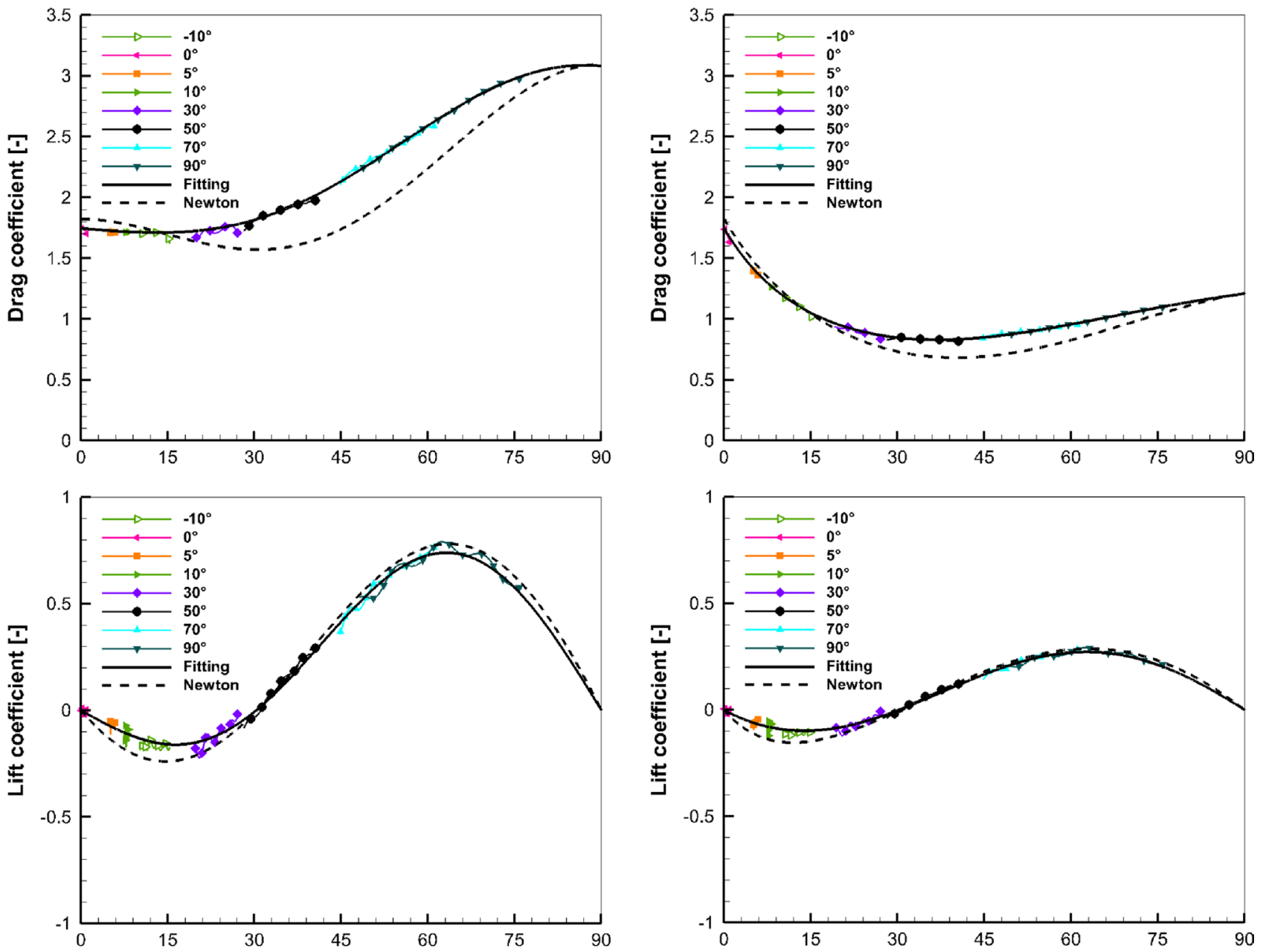

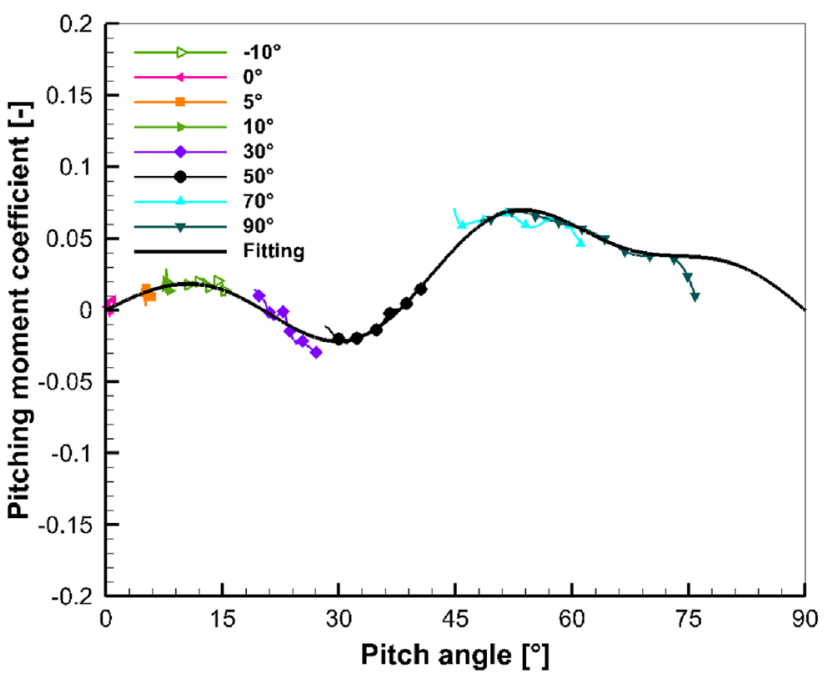

(a)

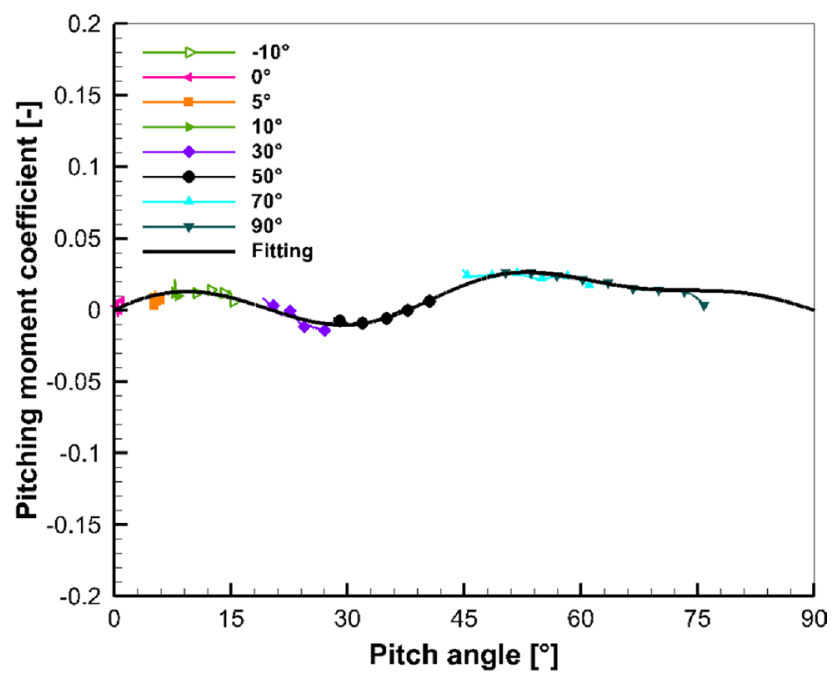

(b)

Fig. 12 Influence of the pitch angle on aerodynamic coefficients based on experimental data at different initial pitch angles determined with different reference areas: (a) circular base area $\left(S_{\text {ref }}=\pi / 4 d^{2}\right)$ and (b) projected frontal area $\left(S_{\text {ref }}=f(d, l, \vartheta)\right)$ 
a pitch-angle-dependent projected frontal area (Fig. 12b) are shown here. The second reference quantity is calculated by means of Eq. (2). Figure 12 reports derived measurement data just in a positive range of pitch angle, where open symbols indicate mirrored values. In doing so, the absolute pitch angles are applied here as well as the lift force and pitching moment coefficients experience a change of sign for the mirrored values. In general, the aerodynamic coefficients reveal a significant dependence on the body orientation, which confirms previous findings of cubes in the literature (Seltner et al. 2019; Hansche and Rinehart 1952).

$S_{\text {ref }}(\vartheta)=\frac{\pi}{4} d^{2}|\cos (\vartheta)|+l d|\sin (\vartheta)|$

Due to the mirror symmetry of cylinders, the correlations between aerodynamic coefficients and pitch angle have a period of $180^{\circ}$. Thus, the results of pitch angles in the range from $0^{\circ}$ to $180^{\circ}$ can be extrapolated on its full-rotation aerodynamic characteristics. Trigonometric functions by means of Fourier series with period $\pi$ are used for the curve fitting on the experimental data. This means that the angular frequency of each Fourier term is an integer multiple of 2. For high-order terms, the Fourier coefficients are very small excepting for the pitching moment coefficient, which is why these terms are neglected for drag and lift coefficients. The resulting functions of drag, lift and pitching moment coefficient are given in Eqs. (3-5) being subject to the pitch angle. The overall uncertainties as described in Sect. 2.2.4 are 2\% in $C_{D}, 11 \%$ in $C_{L}$ and $25 \%$ in $C_{M}$ referred to the peak value.

$$
\begin{aligned}
& C_{D}(\vartheta)=2.273-0.062 \sin (4 \vartheta)+\sum_{i=1}^{2} b_{i} \cos (2 i \vartheta) ; \\
& b_{1}=-0.667, b_{2}=0.141 \\
& C_{L}(\vartheta)=\sum_{i=1}^{2} a_{i} \sin (2 i \vartheta) ; \\
& a_{1}=0.416, a_{2}=-0.424 \\
& C_{M}(\vartheta)=\sum_{i=1}^{6} a_{i} \sin (2 i \vartheta) ; \\
& a_{1}=0.034, a_{2}=-0.030, a_{3}=0.003, a_{4}=0.017, \\
& a_{5}=0.012, a_{6}=-0.006
\end{aligned}
$$

The drag coefficient of cylinders based on circular reference area (see Fig. 12a) exhibits a much greater influence of the inclination angle than of cubes as shown in Seltner et al. (2018). Increasing the pitch angle of the cylindrical body causes first a slight decrease in drag coefficient until $13^{\circ}$ before it begins to increase significantly reaching the peak value at roughly $90^{\circ}$. At a pitch angle of $0^{\circ}$, the drag coefficient is 1.747 , which differs by $5.9 \%$ from the value $\left(C_{D}=1.65\right)$ determined by Hoerner (1965). This underestimation of the base-exposed cylinder's drag can be explained by the neglect of three-dimensional flowfield phenomena, and hence, the aerodynamic influence of the body ends. As opposed to this, the experimental drag coefficient at a pitch angle of $90^{\circ}$ is identical to Hoerner's value $\left(C_{D}=3.08\right.$ referred to the base area), whereas it is overestimated by Penland (1954) $\left(C_{D}=3.16\right)$ having a relative deviation of $2.5 \%$. In comparison, the drag coefficient determined by the modified Newtonian theory decreases more strongly with increasing pitch angle reaching the minimum at a higher pitch angle of $31^{\circ}$ before it begins to increase until $90^{\circ}$, as well. Moreover, the base-exposed drag coefficient is overestimated with a relative deviation of $4.4 \%$ compared to the experimental value, while the side-exposed drag coefficient is almost identical with a relative deviation of $0.5 \%$. Notwithstanding, the hypersonic approximation shows a fairly good agreement with the experimental results.

Asymmetric flowfields in consequence of the body inclination effects the development of lift forces and pitching moments, whose function graphs behave sinusoidally. In Fig. 12a, lift coefficient's maximum of 0.740 appears at a pitch angle of $63^{\circ}$ and its minimum of -0.161 occurs at a pitch angle of $16^{\circ}$. The maximum arises at an angle, where the space diagonal of the cylinder is orthogonal to the inflow direction, and thus, the projected area is close to highest value. Referring to the circular base area, the maximum lift coefficient amounts to $24.0 \%$ of the maximum drag coefficient at $\vartheta=90^{\circ}$. The modified Newtonian theory reveals a good agreement with the experimental results on lift coefficients, whereby the maximum absolute deviation is 0.086 $\left(\vartheta=12^{\circ}\right)$. The graph of pitching moment coefficient has three zero-crossings within the half period at $0^{\circ}, 20^{\circ}$ and $38^{\circ}$, while its maximum occurs at $54^{\circ}$. Surprisingly, positive pitching moment coefficients occur for small positive pitch angles, even though negative values are expected due to a shifting of the stagnation point in counterwise direction. However, the surface pressures of the bottom shell surface are much higher than of the upper side due to a higher recompression of the flow in combination with a stronger reattachment shock (see Fig. 7b), whereby its center of pressure (CoP) lies ahead of the CoG close to the leading edge. This aerodynamic moment acts in opposite direction of rotation than the moment of the stagnation region, whereby the lever arm of the shell surface's CoP is longer than of the stagnation point. By rising the pitch angle of the cylinder, the CoP shifts backward causing a decrease of its lever arm length and aerodynamic moment, whereas both increase for the stagnation point. At a pitch angle of $20^{\circ}$, the moment proportions are in equilibrium $\left(C_{M}=0\right)$, which is not expected for an asymmetric flowfield. Thus, the aerodynamic moment 
proportion of the base behaves contrary to the one of the shell surface explaining zero-crossings for inclined configurations. Analytical outcomes of $C_{M}$ are not presented in Fig. 12, because the pitching moment is constant zero owing to the symmetry of the surface pressure distribution with respect to the xy-, xz- and yz-plane. These results offer vital evidence for the occurrence of significant lift forces and pitching moments of inclined cylinders in hypersonic flowfield, which are remarkable that the impact on rigid body motion should be considered. In addition, there is a good probability that the zero-crossings slightly depend on cylinder's aspect ratio $(l / d)$.

It was shown previously in this analysis that an increasing pitch angle toward $90^{\circ}$ results in an increase in the drag coefficient when using a constant reference area for normalization. However, the frontal area also increases, which has a major influence on the rise of drag coefficient, in fact. By referring to an inclination-dependent reference area as in Fig. 12b, the drag coefficient decreases between a pitch angle of $0^{\circ}$ and $37^{\circ}$ before it begins to increase with lower gradients reaching a local maximum of 1.210 at $90^{\circ}$. This drag coefficient based on the projected reference area amounts just to $39.3 \%$ of the value with constant base reference area, which is why the larger proportion is attributed to the increase in the frontal area. The global maximum occurs at a pitch angle of $0^{\circ}$ and is significantly higher with a relative difference of $44.4 \%$ than the local maximum at roughly $90^{\circ}$. The minimum is reached with a value of 0.831 at an inclination, where the stagnation point is close to cylinder's leading edge. It follows that the part of the body, which protrudes into the flow, primarily determines the magnitude of drag coefficient, whereby the flat-faced configuration (e.g., $\vartheta=0^{\circ}$ ) exhibits a higher effective drag coefficient than the round-curved configuration (e.g., $\vartheta=90^{\circ}$ ) and a much higher effective drag coefficient than the leading-edge configuration (e.g., $\vartheta=37^{\circ}$ ). Thereby, a lower drag coefficient correlates with a lower stand-off distance of the bow-shock (see Fig. 7). As for the lift force and pitching moment coefficients, the consideration of the pitch-angle-dependent reference area effects a decrease of its values with remaining angles for zero-crossings, in general.

In addition to experimental and analytical results, Fig. 13 depicts the numerically determined aerodynamic coefficients by viscous US3D code and inviscid Cart3D code for comparison purpose. It is notable that both numerical solutions have a very good agreement to each other with a relative deviation in drag coefficient of $1.1 \%$ for the baseexposed cylinder and $0.3 \%$ for the side-exposed cylinder, although the Cart3D solver neglects viscous effects in contrast to US3D. This is consistent with the well-established theory like Hoerner (1965) that the viscous effects of blunt bodies in hypersonic flows are subordinate in contrast to compressibility effects. In general, all three aerodynamic
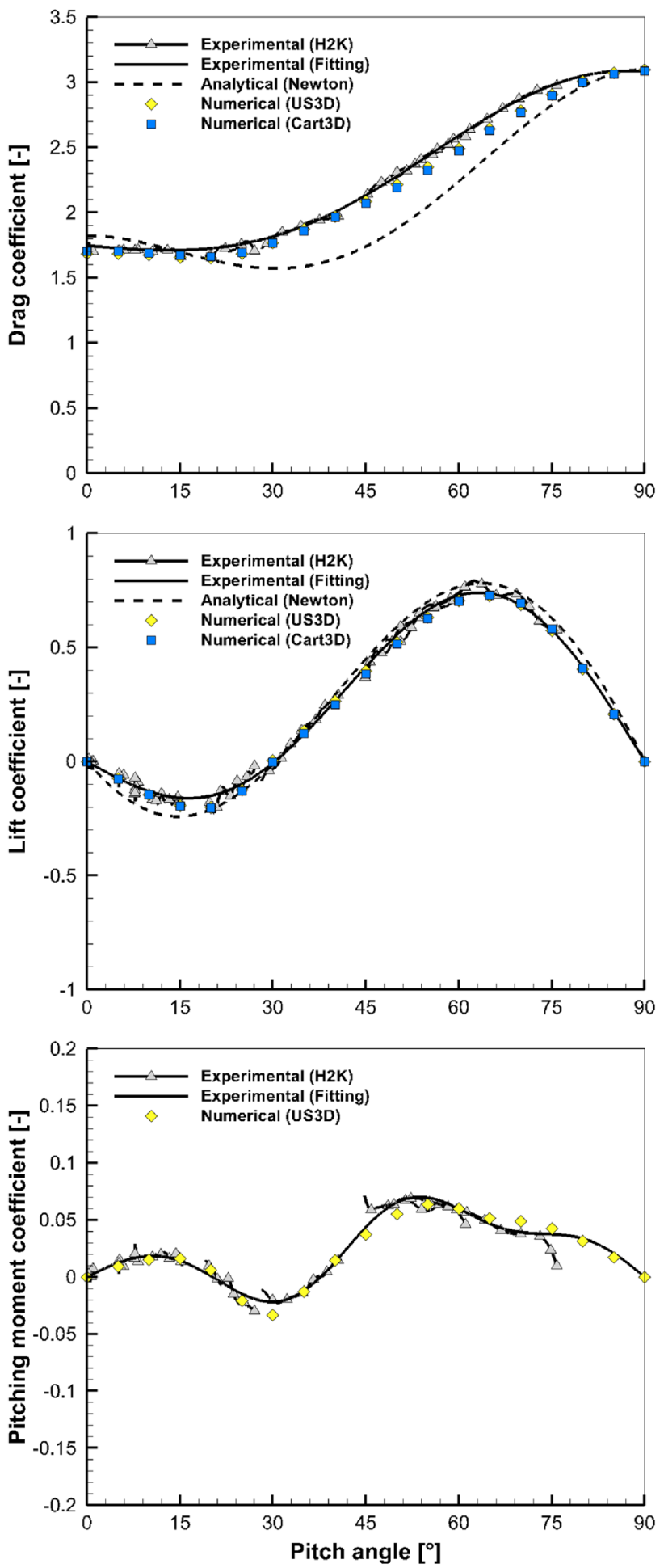

Fig. 13 Comparison of different methods to determine the aerodynamic coefficients as a function of pitch angle

coefficients of both numerical solutions match well with the experimental curves. Yet, the numerical data of the drag coefficient are lower over the almost entire range of pitch angle (except for $90^{\circ}$ ) than the experimental data having 
a deviation based on the US3D data of -0.064 at a pitch angle of $0^{\circ}$ and 0.012 at $90^{\circ}$. It is not surprising here that the numerical and experimental drag coefficients feature a very small deviation, because both flowfields with identical shape and stand-off distance of the bow shock perfectly match with each other at a zero pitch angle (see Fig. 4). As for the lift force coefficient, the results of experiments and corresponding numerical simulations show a very good agreement with zero-crossings at the same pitch angle, where the maximal relative deviation with respect to the peak lift coefficient is very small with $1.3 \%$. By contrast, the agreement between experimental and numerical pitching moment coefficient is good, but the maximal relative deviation with respect to the peak value is higher with a value of $16.7 \%$.

\subsection{Static stability behavior}

Figure 14 summarizes all curves of the curve-fitted aerodynamic coefficients depending on the pitch angle for an entire period of $180^{\circ}$ as well as it depicts the ranges of static stability and trim points with regard to pitching. As requirements for statically stable trim points, moments equilibrium $\left(C_{M}=0\right)$ and negative stability derivative $\left(\partial C_{M} / \partial \vartheta<0\right)$ need to be fulfilled. Three stable trimmed flight attitudes exist at roughly $21^{\circ}, 90^{\circ}$ and $159^{\circ}$ where both conditions are satisfied. Unexpectedly, the base-exposed orientation does not fulfill the stability condition, and in consequence, it is an unstable trimmed attitude, while a flight configuration at a pitch angle of $21^{\circ}$ is a stable trimmed attitude despite an asymmetric flowfield. Thus, the static stability of a cylinder reveals no dependency on the symmetry of flow topology. Both results of CFD simulations and wind tunnel tests confirm these findings.

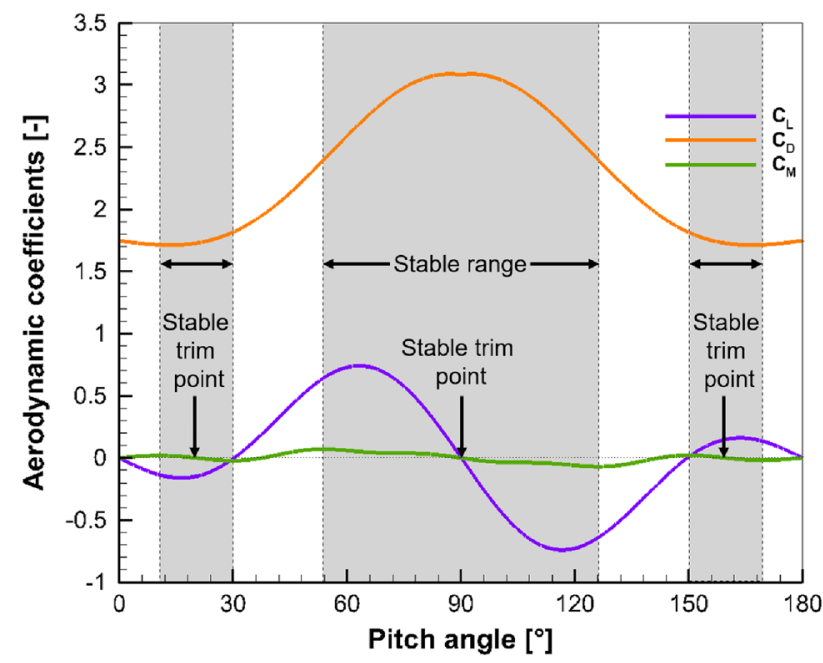

Fig. 14 Static stability depending on pitch angle
The magnitude of stability depends on the amount of the stability derivative. In the neighborhood of $90^{\circ}$, the highest negative stability derivative is obtained with a peak value of $-0.260 \mathrm{rad}^{-1}$, which is the range of highest drag coefficient and hence the highest pressure force. Since one edge of the cylinder moves upstream due to an initiated rotation away from a side-exposed configuration, the stagnation point shifts toward this upstream edge. In consequence, the body part, which protrudes more into the flow, experiences a higher surface pressure causing a reverse pitching moment and hence a counterwise rotation. In addition to the maximal stability at $90^{\circ}$, the pitch angle is statically stable in a wide range from $54^{\circ}$ to $126^{\circ}$, which is why a side-exposed configuration is a probably final flight attitude for randomly tumbling cylinders.

\subsection{Effect of axially symmetrical cavity}

The axially symmetrical cavity as of the dogbone is a significant geometry modification of the shell surface in comparison to the ordinary cylinder, which is why an significant impact on the aerodynamic coefficients is expected. Aerodynamic coefficients based on circular base as well as projected area of the dogbone from present free-flight tests are compared in Fig. 15 with the cylinder's results under the same test conditions. Great differences between both bodies in drag force, lift force and pitching moment coefficient arise as expected (see Fig. 15a), whereby the differences for low pitch angles are small in contrast to higher angles. At a zero pitch angle, the geometry of the flow-exposed forebody, and hence, the shape of the bow shock is the same, which explains the similarity of aerodynamic force coefficients for low pitch angle. However, the experimentally determined drag coefficients toward $90^{\circ}$ apparently diverge with a relative difference, for example, of $27.3 \%$ at $\vartheta=70^{\circ}$ that can be anticipated due to a lower frontal area of the dogbone compared to the cylinder. As for the lift force and pitching moment coefficients, the greatest differences occur in the vicinity of $45^{\circ}$ that complex unsteady flow structures appear in the cavity (see Fig. 16). In comparison with the cylinder, the same flow features like bow shock, expansion fans and wake region with a recompression shock are visible in Fig. 16. By contrast, SSI appears that is the dominant flow phenomenon in the windward part of the cavity-inducing extremely high pressures and heating rates (as described in Edney (1968b)). The bow shock here consists of two parts, whereby the attached shock impinges almost perpendicularly the downstream detached shock in such a way that a type IV shock-shock interaction as in Edney (1968a) emerges (see Fig. 16a) effecting a supersonic jet impingement on the model surface. At the intersection of both shocks, a short-transmitted shock connects both parts of 

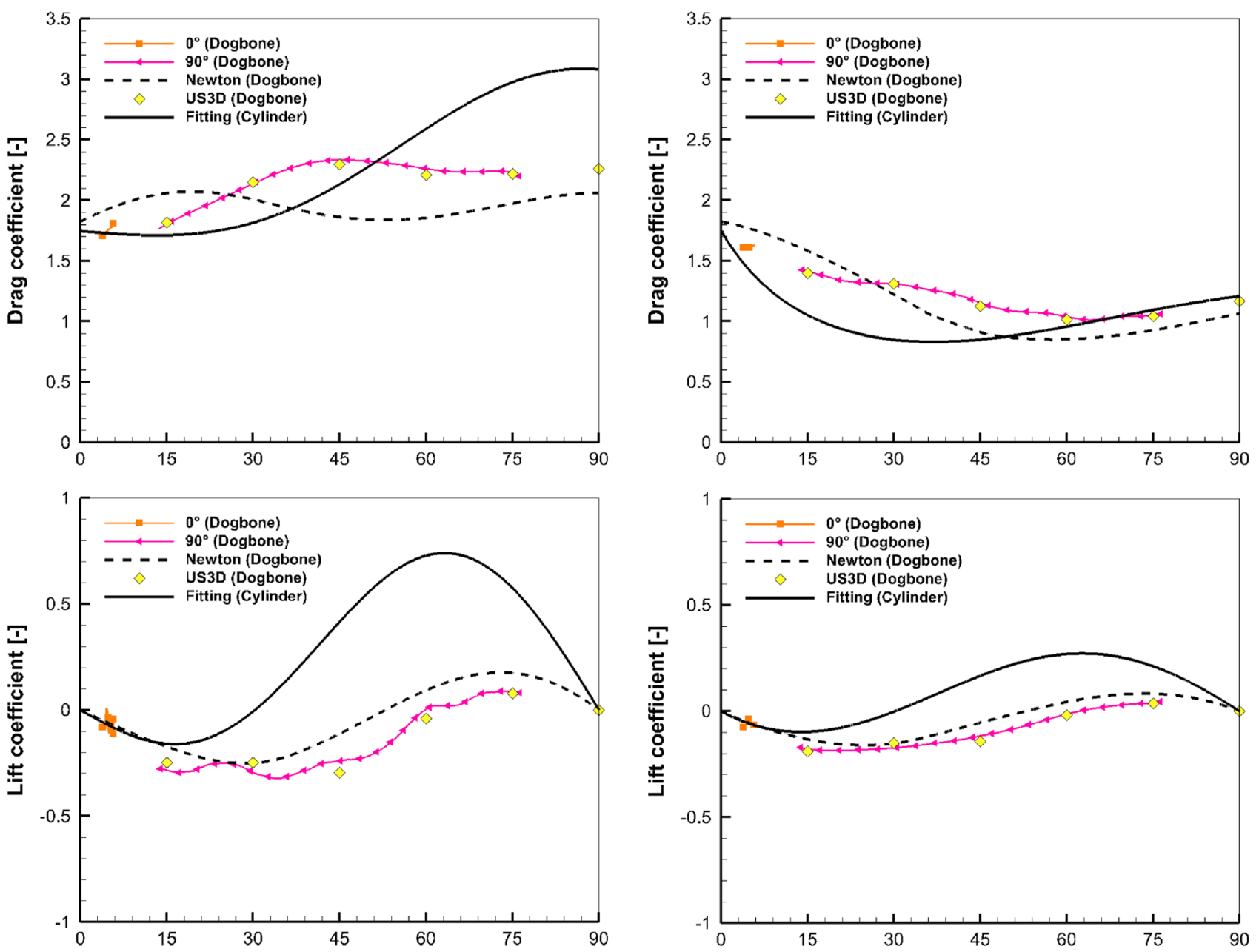

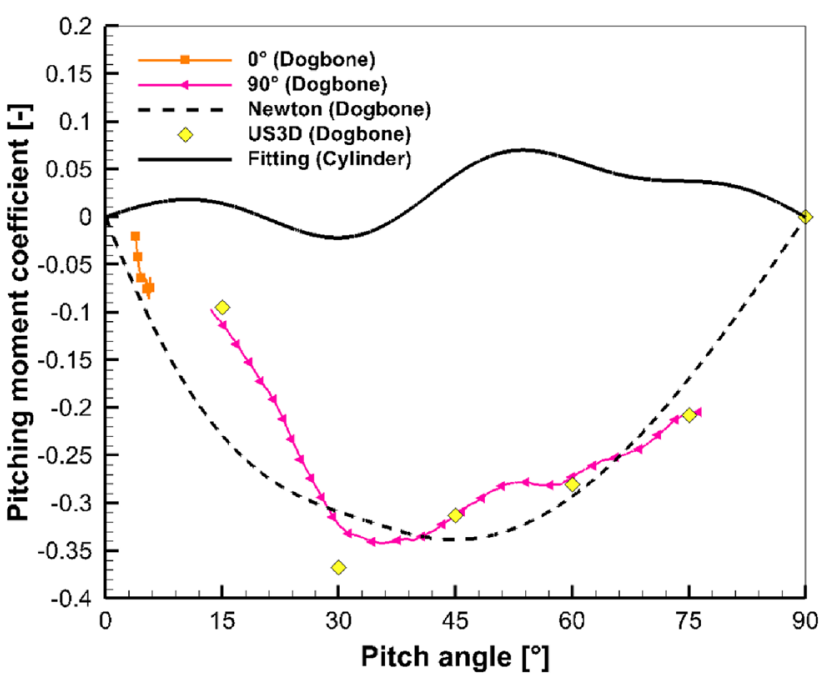

(a)

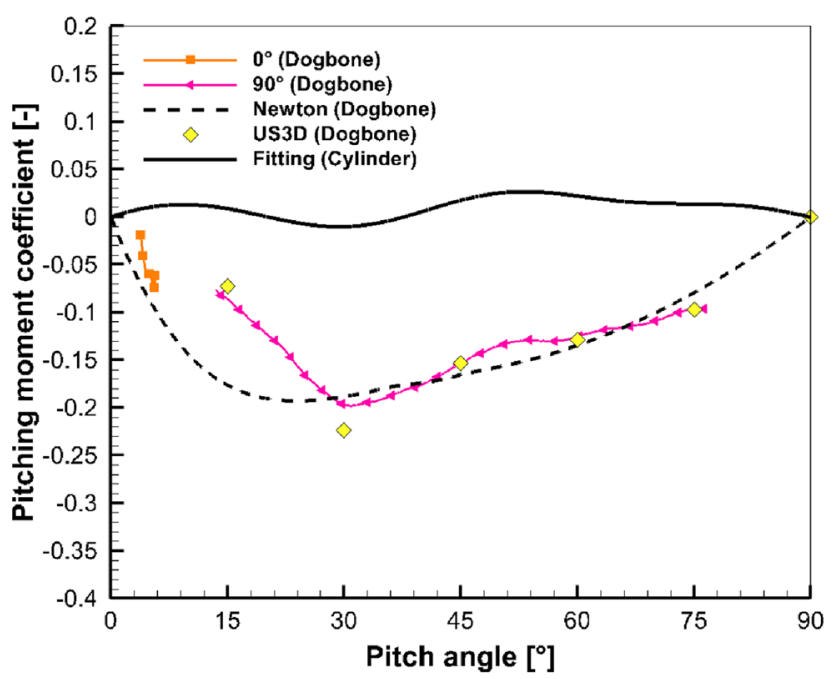

(b)

Fig. 15 Comparison of dogbone's and cylinder's aerodynamic coefficients based on different reference areas: a circular base area $\left(S_{\text {ref }}=\pi / 4 \mathrm{~d}^{2}\right)$ and $\mathbf{b}$ projected frontal area $\left(S_{\text {ref }}=f(d, l, \vartheta)\right)$ 

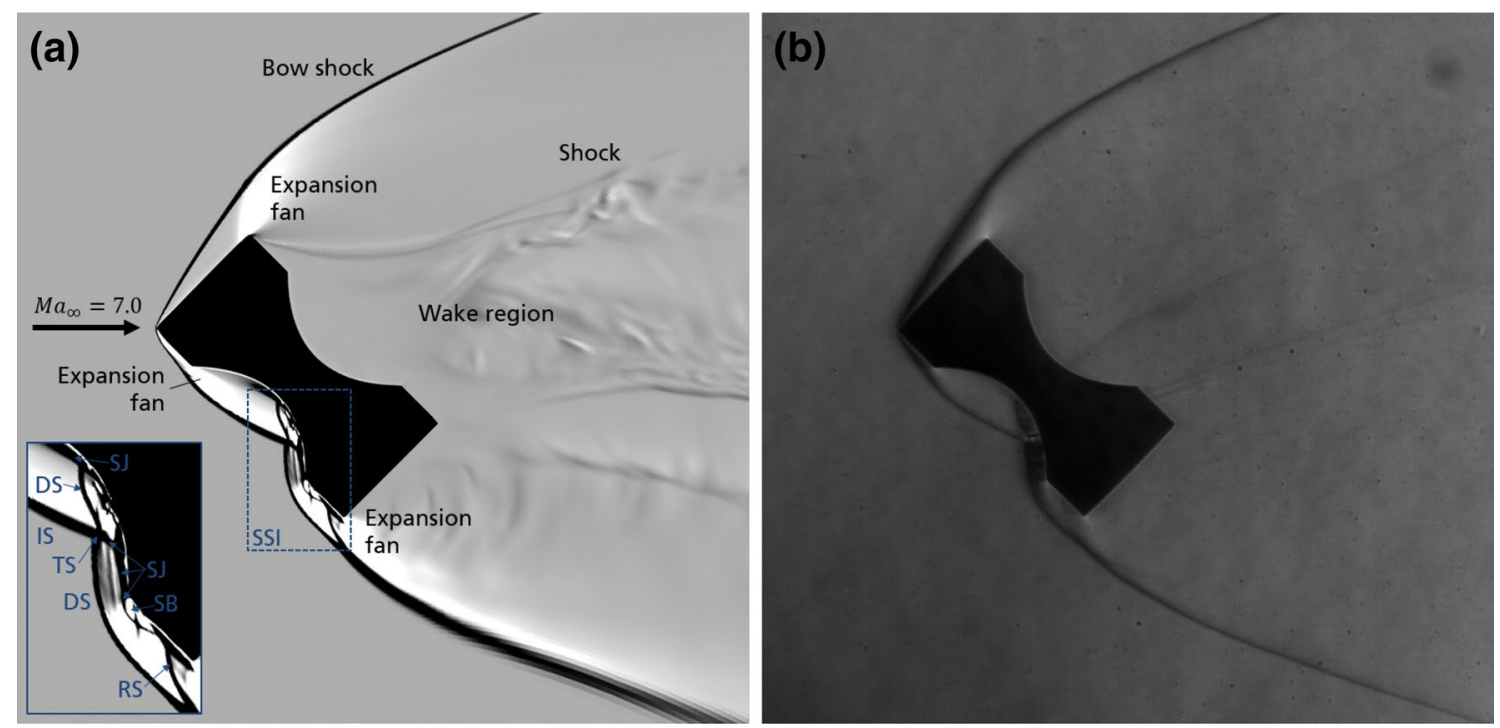

Fig. 16 Comparison of (a) numerical and (b) experimental flow field around a dogbone at $\vartheta=45^{\circ}$ including description of flow structures with impingement shock (IS), detached shock (DS), transmitted shock (TS), supersonic jet (SJ), separation bubble (SB) and reattachment shock (RS)

the detached shock, whereby a supersonic jet as an extension of the impingement shock also forms downstream of this transmitted shock. The supersonic jet flows toward the model surface as it separates two regions of subsonic flow. Close to the impingement point, the supersonic jet is primarily deflected outward and follows the contour until the edge of the transition to the cylindrical part, where it separates and reattaches shortly after. Thus, a separation bubble develops underneath the jet causing a downstream reattachment shock. This shock impinges the detached shock provoking a type VI shock-shock interaction. In conclusion, the region of peak surface pressures due to supersonic jet impingement seems to be reasonable for the shown differences in the aerodynamic coefficients between cylinder and dogbone. On the one hand, this peak pressure on the lower surface causes a great negative pitching moment. On the other hands, the surface's normal vectors close to the impingement are almost parallel to the inflow direction, which is why the resulting pressure force consists mainly of a drag proportion instead of a lift proportion in comparison to the cylindrical body without cavity.

It is crucial to note that the numerical aerodynamic coefficients of the dogbone determined by US3D reveal a good agreement with the experimental data as shown in Fig. 15. The maximum absolute deviations here are very low with 0.061 in the drag coefficient and with 0.054 in the lift coefficient both at $\vartheta=45^{\circ}$. Yet, a single significant deviation can be observed in the pitching moment coefficient at a pitch angle of $30^{\circ}$, which is in the uncertainty margin of both values. For this configuration, the impingement as a result of SSI is close to the interface between cavity and cylindrical part.
Furthermore, dogbone's hypersonic approximation determined by modified Newtonian flow theory is shown in Fig. 15, as well. At the first glance, the experimental and analytic data of the dogbone reveal a fairly good agreement. In particular, the approximations of lift force and pitching moment coefficient are consistent with the results of the free-flight tests having a maximum absolute deviation in lift coefficient of 0.174 (see Fig. 15a). The analytical drag coefficient shows a good consistency with the order of magnitude compared to the experimental data but a different development with respect to the body inclination. Thereby, the approximated drag coefficient starts to increase at a smaller pitch angle with increasing inclination reaching the maximum at $19^{\circ}$ before it experiences an unexpected alternation of decreasing and increasing. The larger deviations in the aerodynamic coefficients can be explained by the neglect of SSI effects on surface pressure distribution by the use of the Newtonian flow theory.

Interestingly, the course of pitching moment coefficient in Fig. 15 is considerably different between the cylindrical body with and without cavity, whereby the present cavity causes a continuously negative pitching moment over the entire range of $90^{\circ}$ with a roughly 5 times higher absolute peak value of the dogbone than of the cylinder. In contrast to two stable plus two unstable trim points of the cylinder in the range between $0^{\circ}$ and $90^{\circ}$, the dogbone merely has one stable and one unstable trim point. These two trim points of the dogbone are at the same pitch angle as of the cylinder but with a contrary stability behavior. The most remarkable result from these data is that the present axially symmetrical cavity effects a reversal of the static stability behavior at a zero pitch angle with considerable magnitude by contrast 
with the body without cavity. Thereby, the dogbone's stability derivative of $-1.172 \mathrm{rad}^{-1}$ at a pitch angle of $0^{\circ}$ is roughly 5 times greater than of the cylinder's peak stability derivative at $90^{\circ}$. As a result, it is more likely that a randomly tumbling cylindrical body with the present axially symmetric cavity tends to a base-exposed configuration as a final flight attitude.

\section{Conclusion}

Rotating cylindrical bodies with and without cavity in Mach-7 flowfield have been studied experimentally and numerically in the present work to research the impact of flight attitude on the aerodynamic behavior. Therefore, free-flight tests were carried out in the $\mathrm{H} 2 \mathrm{~K}$ at the DLR in Cologne, using both non-intrusive stereo-tracking measurement technique to measure high-speed $6 \mathrm{DoF}$ motion data as well as schlieren photography to visualize the flowfield. Motion derivatives including aerodynamic coefficients were determined based on the time-resolved positions and orientation angles of the motion data by means of an extensive post-processing. NASA's inviscid flow solver Cart3D and compressible Navier-Stokes solver US3D were employed for numerical simulations.

Taken together, the flight attitude of cylindrical bodies in hypersonic flows shows a heavy influence on the gas dynamic flowfield and in consequence the aerodynamic forces and moments just like the cube in Seltner et al. (2019). The numerical results confirm this finding as well as exhibit a good agreement with the experimental outcome. In comparison to the present experimental results, the literature value for cylinders in axial flow by Hoerner (1965) slightly underestimates the aerodynamic drag coefficient, whereas a very good agreement is revealed for cylinders in crossflow. The analytical values determined by modified Newtonian flow theory overestimate the impact of the flight attitude on lift force coefficients and mostly underestimate this impact on drag force coefficients for inclined bodies. Between a pitch angle of $0^{\circ}$ and $90^{\circ}$, the drag coefficient based on a constant base reference area almost doubles. However, this strong rise of $C_{D}$ with increasing pitch angle is primary an effect of rising effective aerodynamic front face. As a consequence, the course of drag coefficient based on a pitch-angle-dependent frontal reference area significantly changes compared to a constant reference area, that the maximum value of 1.747 appears for a cylinder in axial flow $\left(\vartheta=0^{\circ}\right)$. It is notable that the pitching moment coefficient of the cylindrical body without cavity is characterized by unforeseeable points of extrema as well as an unexpected static stability behavior. In doing so, the cylinder in crossflow $\left(\vartheta=90^{\circ}\right)$ is statically stable, whereas the cylinder in axial flow $\left(\vartheta=0^{\circ}\right)$ behaves statically unstable. Furthermore, the including of an axially symmetrical cavity shows the presence of complex unsteady flow structures like a type IV shock-shock interaction within the windward cavity region. The current study reveals the evidence that this cavity causes a considerable change of the aerodynamic coefficients in comparison with the ordinary cylinder. Regarding the static stability behavior, it has been found here that the cylindrical body becomes statically stable due to the cavity at a zero pitch angle. To sum up, an implication of this paper is the possibility to consider the impact of the attitude on the flight path and velocity of cylindrical bodies for atmospheric re/entry analysis.

However, a small number of potential weaknesses of the current study need to be considered. First, the aerodynamic coefficients from free-flight tests of the cylindrical body with and without cavity exhibit a lack of data in some ranges of the pitch angle. However, these incomplete experimental data are supplemented by numerical data by Cart3D and US3D. Second, the first and last values of motion derivatives are partially influenced during the filtering of the post-processing by distorted motion data coming from measurement values for that parts of the test object are in the inhomogenous shear layer of H2K's free jet. To weaken this effect, it is planned to develop an new post-processing algorithm, whereby the aerodynamic coefficients should be determined by fitting an analytic trajectory, as a result of the motion equations, on the measured trajectory. Third, just a simple error analysis based on statistical errors of regression is applied in the present paper, but a detailed error propagation approach based on a majority of uncertainty sources is already in progress.

Finally, these findings suggest the following opportunities for future research. On the one hand, the investigation of cylinder's aspect ratio is of interest concerning the effect on the aerodynamic behavior and hence the resulting flight trajectories. On the other hands, parameter studies on the design of axially symmetrical cavities would help to understand how the stability behavior of cylindrical bodies can be influenced by shape, position and dimensions of cavities.

Supplementary Information The online version contains supplementary material available at https://doi.org/10.1007/s00348-021-03269-6.

Acknowledgements The present work has been performed within the framework of the Program Helmholtz Research School on Security Technologies (HRSST) and benefited from a funding by the DLR program directorate Defence \& Security. Support was also given by NASA's Planetary Defense Coordination Office (PDCO) managed by Mr. Lindley Johnson, who has funded the work of the Asteroid Threat Assessment Project (ATAP). Special thanks also go to Mr. Michael Kosbow and Mr. Marco Schmors for the operation of the H2K facility. 
Funding Open Access funding enabled and organized by Projekt DEAL.

\section{Declarations}

Conflict of interest The authors declare that they have no conflict of interest.

Open Access This article is licensed under a Creative Commons Attribution 4.0 International License, which permits use, sharing, adaptation, distribution and reproduction in any medium or format, as long as you give appropriate credit to the original author(s) and the source, provide a link to the Creative Commons licence, and indicate if changes were made. The images or other third party material in this article are included in the article's Creative Commons licence, unless indicated otherwise in a credit line to the material. If material is not included in the article's Creative Commons licence and your intended use is not permitted by statutory regulation or exceeds the permitted use, you will need to obtain permission directly from the copyright holder. To view a copy of this licence, visit http://creativecommons.org/licenses/by/4.0/.

\section{References}

Achner M (2011) TMK Technische Notiz 04. Technical report TMKTN04, DLR, Köln

Aftosmis MJ, Berger MJ, Melton JE (1998) Robust and efficient Cartesian mesh generation for component-based geometry. AIAA J 36(6):952-960. https://doi.org/10.2514/2.464

Aftosmis MJ, Berger MJ, Adomavicius G (2000) A parallel multilevel method for adaptively refined Cartesian grids with embedded boundaries. In: 38th Aerospace sciences meeting and exhibit, american institute of aeronautics and astronautics (AIAA), Reno. https://doi.org/10.2514/6.2000-808

Beberniss TJ, Ehrhardt DA (2017) High-speed 3D digital image correlation vibration measurement: recent advancements and noted limitations. Mech Syst Signal Process 86:35-48. https://doi.org/ 10.1016/j.ymssp.2016.04.014

Daub D, Esser B, Gülhan A (2020a) Experiments on high-temperature hypersonic fluid-structure interaction with plastic deformation. AIAA J 58(4):1423-1431. https://doi.org/10.2514/1.J059150

Daub D, Willems S, Esser B, Gülhan A (2020b) Experiments on aerothermal supersonic fluid-structure interaction, chap 21. In: Adams NA, Schrder W, Radespiel R, Haidn OJ, Sattelmayer T, Stemmer C, Weigand B (eds) Future space-transport-system components under high thermal and mechanical loads, notes on numerical fluid mechanics and multidisciplinary design, vol 146, 1st edn. Springer International Publishing, Berlin, pp 323-339. https://doi.org/10. 1007/978-3-030-53847-7_21

Edney B (1968a) Anomalous heat transfer and pressure distributions on blunt bodies at hypersonic speeds in the presence of an impinging shock. Techreport FFA Report 115, The Aeronautical Research Institute of Sweden, Stockholm. https://www.osti.gov/servlets/ purl $/ 4480948$

Edney B (1968b) Effects of shock impingement on the heat transfer around blunt bodies. AIAA J 6(1):15-21. https://doi.org/10. $2514 / 3.4435$

ESA Space Debris Office (2020) ESA's annual space environment report. Techreport GEN-DB-LOG-00288-OPS-SD, ESA Space Operations. Centre (ESOC), Darmstadt

Gowen FE, Perkins EW (1953) Drag of circular cylinders for a wide range of Reynolds numbers and Mach numbers. Technical report,
Ames Aeronautical Laboratory, Moffett Field, California. https:// ntrs.nasa.gov/archive/nasa/casi.ntrs.nasa.gov/19930087134.pdf

Grossir G, Puorto D, Ilich Z, Paris S, Chazot O, Rumeau S, Spel M, Annaloro J (2020) Aerodynamic characterization of space debris in the VKI Longshot hypersonic tunnel using a free-flight measurement technique. Exp Fluids 61(7). https://doi.org/10.1007/ s00348-020-02995-7

Hansche GE, Rinehart JS (1952) Air drag on cubes at Mach numbers 0.5 to 3.5. J Aeronaut Sci 19(2):83-84. https://doi.org/10.2514/8. 2166

Hoerner SF (1965) Fluid-dynamic drag: Practical information on aerodynamic drag and hydrodynamic resistance. Hoerner Fluid Dynamics, 2nd edn, Bakersfield, California

Kaufman LG II, Meckler L, Hartofilis SA (1966) An investigation of flow separation and aerodynamic controls at hypersonic speeds. J Aircr 3(6):555-561. https://doi.org/10.2514/3.43776

Laurence SJ, Parziale NJ, Deiterding R (2012) Dynamical separation of spherical bodies in supersonic flow. J Fluid Mech 713:159-182. https://doi.org/10.1017/jfm.2012.453

Lee S, Song H, Park G (2017) Freefalling heated sphere in a shock tunnel. AIAA J 55(11):3995-3998. https://doi.org/10.2514/1. J055967

Lees L (2003) Hypersonic flow. J Spacecr Rocket 40(5):700-735. https://doi.org/10.2514/2.6897

Marwege A, Willems S, Gülhan A, Aftosmis MJ, Stern EC (2018) Superposition method for force estimations on bodies in supersonic and hypersonic flows. J Spacecr Rocket 55(5):1166-1180. https://doi.org/10.2514/1.A34128

Maslach GJ, Schaaf SA (1962) Cylinder drag in the transition from continuum to free molecule flow. Technical report, Univeristy of California. http://www.dtic.mil/dtic/tr/fulltext/u2/274385.pdf

Matthews RK, Eaves RH Jr (1967) Comparison of theoretical and experimental pressure and heat transfer distributions on three blunt nosed cylinders in hypersonic flow. Technical Report AEDCTR-67-148, Arnold Engineering Development Center, Arnold Air Force Station, Tennessee. https://apps.dtic.mil/sti/ pdfs/AD0819547.pdf

Nemec M, Aftosmis MJ (2016) Toward automatic verification of goal-oriented flow simulations. In: Mehta UB, Eklund DR, Romero VJ, Pearce JA, Keim NS (eds) Simulation credibility: Advances in verification, validation, and uncertainty quantification, 1st edn. National Aeronautics and Space Administration (NASA), chap 4, pp 115-156. https://ntrs.nasa.gov/citations/ 20160013550

Niezgodka FJ (2001) Der Hyperschallwindkanal H2K des DLR in Köln-Porz (Stand 2000). Technical report, DLR, Köln. https:// elib.dlr.de/13874

Nompelis I, Drayna T, Candler G (2005) A parallel unstructured implicit solver for hypersonic reacting flow simulation. In: 17th AIAA Computational Fluid Dynamics Conference, American Institute of Aeronautics and Astronautics (AIAA), Toronto. https://doi.org/10.2514/6.2005-4867

Park SH, Park G (2020) Separation process of multi-spheres in hypersonic flow. Adv Space Res 65(1):392-406. https://doi.org/10. 1016/j.asr.2019.10.009

Penland JA (1954) Aerodynamic characteristics of a circular cylinder at Mach number 6.86 and angles of attack up to $90^{\circ}$. Technical report, Langley Aeronautical Laboratory. https://ntrs.nasa.gov/ archive/nasa/casi.ntrs.nasa.gov/19930093717.pdf

Preci A, Gülhan A, Clopeau E, Tran P, Ferracina L, Marraffa L (2015) Dynamic characteristics of MarcoPolo-R entry capsule in low subsonic flow. CEAS Space J 8:23-33. https://doi.org/10.1007/ s12567-015-0105-1

Rees TW, Fisher TB, Bruce PJK, Merrifield JA, Quinn MK (2020) Experimental characterization of the hypersonic flow 
around a cuboid. Exp Fluids 61(7). https://doi.org/10.1007/ s00348-020-02975-x

Register PJ, Aftosmis MJ, Stern EC, Brock JM, Seltner PM, Willems S, Gülhan A, Mathias DL (2020) Interactions between asteroid fragments during atmospheric entry. Icarus 337(113468). https:// doi.org/10.1016/j.icarus.2019.113468

Riley ZB, Perez RA, Bartram GW, Spottswood SM, Smarslok BP, Beberniss TJ (2019) Aerothermoelastic experimental design for the AEDC/VKF tunnel C: challenges associated with measuring the response of flexible panels in high-temperature, high-speed wind tunnels. J Sound Vib 441:96-105. https://doi.org/10.1016/j. jsv.2018.10.022

Rouse H (1946) Elementary mechanics of fluids, 1st edn. Dover Publications, New York

Seltner PM, Willems S, Gülhan A (2018). Experimental determination of aerodynamic coefficients of simple-shaped bodies free-flying in hypersonic flow. In: First international conference on high-speed vehicle science technology (HiSST), council of european aerospacesocieties (CEAS), Moscow

Seltner PM, Willems S, Gülhan A (2019) Aerodynamic coefficients of free-flying cubes in hypersonic flowfield. J Spacecr Rocket 56(6):1725-1734. https://doi.org/10.2514/1.a34345
Spalart P, Allmaras S (1992) A one-equation turbulence model for aerodynamic flows. In: 30th Aerospace sciences meeting and exhibit, american institute of aeronautics and astronautics (AIAA), Reno. https://doi.org/10.2514/6.1992-439

Subbareddy PK, Candler GV (2009) A fully discrete, kinetic energy consistent finite-volume scheme for compressible flows. J Comput Phys 228(5):1347-1364. https://doi.org/10.1016/j.jcp.2008. 10.026

Vennard JF (1940) Elementary fluid mechanics, 1st edn. Wiley, New York

Wright MJ, Candler GV, Bose D (1998) Data-parallel line relaxation method for the Navier-Stokes equations. AIAA J 36(9):16031609. https://doi.org/10.2514/2.586

Publisher's Note Springer Nature remains neutral with regard to jurisdictional claims in published maps and institutional affiliations.

\section{Authors and Affiliations}

\section{Patrick M. Seltner ${ }^{1}\left[\right.$. Sebastian Willems ${ }^{1} \cdot$ Ali Gülhan $^{1} \cdot$ Eric C. Stern ${ }^{2} \cdot$ Joseph M. Brock $^{3} \cdot$ Michael J. Aftosmis $^{4}$}

Eric C. Stern

eric.c.stern@nasa.gov

1 Supersonic and Hypersonic Technologies Department, German Aerospace Center (DLR), Institute of Aerodynamics and Flow Technology, 51170 Cologne, Germany

2 NASA Ames Research Center, Thermal Protection Materials Branch, Moffett Field, California 94035, USA
3 Analytical Mechanics Associates, Aerothermodynamics Branch, Moffett Field, California 94035, USA

4 NASA Ames Research Center, Computational Aerosciences Branch, Moffett Field, California 94035, USA 